QUARTERLY OF APPLIED MATHEMATICS

VOLUME LXIII, NUMBER 3

SEPTEMBER 2005, PAGES 575-600

S $0033-569 \mathrm{X}(05) 00980-4$

Article electronically published on August 18, 2005

\title{
CONVERGENCE TO EQUILIBRIUM RAREFACTION WAVES FOR DISCONTINUOUS SOLUTIONS OF SHALLOW WATER WAVE EQUATIONS WITH RELAXATION
}

\author{
BY
}

HAITAO FAN (Department of Mathematics, Georgetown University, Washington, DC 20057-1233)

AND

TAO LUO (Department of Mathematics, Georgetown University, Washington, DC 20057-1233)

Abstract. The purpose of this paper is to study the discontinuous solutions to a shallow water wave equation with relaxation. The typical initial value problem of discontinuous solutions is the Riemann problem. Unlike the homogeneous hyperbolic conservation laws, due to the inhomogeneity of the system studied here, the solutions of the Riemann problem do not have a self-similar structure anymore. This problem can be formulated as a free boundary problem. We show that the Riemann solutions still have a piecewise smooth structure globally and converge to the rarefaction waves of the equilibrium equation as time tends to infinity.

1. Introduction. The propagation of surface shallow water waves in a river with constant inclined bottom topography can be described in Eulerian coordinates by the following system of hyperbolic conservation laws with a relaxation term:

$$
\left\{\begin{array}{l}
h_{\tau}+(h u)_{\xi}=0, \\
(h u)_{\tau}+\left(h u^{2}+\frac{1}{2} g^{\prime} h^{2}\right)_{\xi}=g^{\prime} h S-C_{f} u^{2} .
\end{array}\right.
$$

The assumptions of hydrostatic pressure distribution, gravitational effect, and frictional bottom topography are used in deriving the shallow water river model (cf. 42]). In this case, $g^{\prime}=g \cos \alpha, S=\tan \alpha$, with $0<\alpha<\pi / 2, g$ is the gravitational acceleration, $\alpha$ is a constant representing the inclination angle of the river, $C_{f}>0$ is the constant frictional coefficient, $h>0$ and $u>0$ are the depth and velocity of the water respectively, and $\tau$ and $\xi$ are the time and space variables, respectively.

Received February 10, 2005.

2000 Mathematics Subject Classification. Primary 35L65, 35L67, 35L60.

Key words and phrases. Shallow water wave equations, relaxation, shock waves, rarefaction waves, free boundary problem.

E-mail address: fanh@georgetown.edu

E-mail address: t148@georgetown.edu

(C)2005 Brown University 
Since it is more convenient to consider system (1.1) in Lagrangian coordinates, we use the usual transformation $x=\int_{\xi(\tau)}^{\xi} h(y, \tau) d y$ and $t=\tau$, where $\xi(\tau)$ is an arbitrary particle path satisfying $\dot{\xi}(\tau)=u(\xi(\tau), \tau)$. Under this transformation, system (1.1) becomes

$$
\left\{\begin{array}{l}
v_{t}-u_{x}=0 \\
u_{t}+p(v)_{x}=g^{\prime} S-C_{f} u^{2} v
\end{array}\right.
$$

where $v=1 / h$, and $p(v)=\frac{1}{2} g^{\prime} v^{-2}$. This system is strictly hyperbolic when $0<v<$ $\infty$ with two distinct characteristic speeds $\lambda_{1}(v)=-\sqrt{-p^{\prime}(v)}=-\sqrt{g^{\prime}} v^{-3 / 2}, \lambda_{2}(v)=$ $\sqrt{-p^{\prime}(v)}=\sqrt{g^{\prime}} v^{-3 / 2}$, and two Riemann invariants

$$
w(u, v)=u+m(v), \quad z(u, v)=u-m(v),
$$

with $m(v)=-2 \sqrt{g^{\prime} / v}$ satisfying $m^{\prime}(v)=\lambda_{2}(v)$. When inertia is neglected, the relaxation term $g^{\prime} S-C_{f} u^{2} v$ vanishes, the system is in "equilibrium" and the "equilibrium" equation corresponding to (1.2) is given by

$$
v_{t}-f(v)_{x}=0,
$$

where $f(v)= \pm \sqrt{\frac{g^{\prime} S}{C_{f} v}}$ satisfies $g^{\prime} S-C_{f}(f(v))^{2} v=0$. In the following, we consider the case when $(v, u)$ is in a small neighborhood of a point on the "equilibrium" curve $u=\sqrt{\frac{g^{\prime} S}{C_{f} v}}$, i.e., $f(v)=\sqrt{\frac{g^{\prime} S}{C_{f} v}}$. It is expected that system (1.2), as $t \rightarrow \infty$, is well approximated by the "equilibrium" equation (1.4), provided the subcharacteristic condition $\left|f^{\prime}(v)\right|<\sqrt{-p^{\prime}(v)}$ holds. This subcharacteristic condition serves as the stability condition (see [42] and [22]), and it turns out to be very simple in the present situation, i.e.,

$$
\tan \alpha=S<4 C_{f},
$$

which means the inclination angle is less than a critical value.

Previous works on nonlinear hyperbolic systems with relaxation are mainly on smooth solutions with small derivatives (cf. [22], 50], [10], [54] and [17]). In general, if the derivatives of initial data are not small, discontinuities will develop in finite time. Therefore, it is quite natural to study discontinuous solutions. For water waves, discontinuities satisfying the Rankine-Hugoniot condition represent turbulent bores, or "hydraulic jumps" in water wave theory (cf. 42]).

The simplest problem with discontinuous initial data is the Riemann problem, i.e., the initial value problem of (1.2) with the following initial data:

$$
(v(x, 0), u(x, 0))= \begin{cases}\left(v_{l}, u_{l}\right), & -\infty<x<0, \\ \left(v_{r}, u_{r}\right) & 0<x<+\infty .\end{cases}
$$

The Riemann solutions play an important role in the study of quasi-linear hyperbolic systems. It is very important and interesting to understand the structure of Riemann solutions in the study of hyperbolic conservation laws, since the Riemann solutions serve as building blocks of general discontinuous solutions and the standard test model of mathematical theories and numerical methods for solving nonlinear hyperbolic systems. Also, the Riemann solutions may determine the large-time behavior of general discontinuous solutions. Moreover, for system (1.2), the current techniques are inadequate to give a 
good understanding of the structure and behavior of general discontinuous solutions of system (1.2). Of course the Riemann solutions of homogeneous quasilinear hyperbolic systems of the form $U_{t}+F(U)_{x}=0$ are well understood (cf. 35]). For a homogeneous system, since the system and the Riemann initial data are invariant under the uniform stretching of coordinates $(x, t) \rightarrow(a x, a t)$, the Riemann solutions admit self-similar solutions of the form $U(x / t)$. Therefore, for homogeneous systems, the Riemann solutions can be given by explicit formulas whenever they exist. However, for inhomogeneous systems, the Riemann solutions lose their self-similar structure, and have much more complicated and richer structures.

In this paper, we assume that the two states $\left(v_{l}, u_{l}\right)$ and $\left(v_{r}, u_{r}\right)$, satisfying $v_{r}>$ $0, v_{l}>0, u_{r}>0, u_{l}>0$, are connected by $S_{1} S_{2}$ in the $(v, u)$ phase plane (see [35]). That is, there exists an intermediate state $\left(v_{m}, u_{m}\right)$ such that $\left(v_{m}, u_{m}\right)$ is connected to $\left(v_{l}, u_{l}\right)$ by a 1 -shock wave, i.e.

$$
u_{m}-u_{l}=-\sqrt{-\left(p\left(v_{m}\right)-p\left(v_{l}\right)\right)\left(v_{m}-v_{l}\right)}, \quad v_{l}>v_{m},
$$

and $\left(v_{r}, u_{r}\right)$ is connected to $\left(v_{m}, u_{m}\right)$ by a 2 -shock wave,

$$
u_{r}-u_{m}=\sqrt{-\left(p\left(v_{m}\right)-p\left(v_{r}\right)\right)\left(v_{m}-v_{r}\right)}, \quad v_{m}<v_{r} .
$$

When $\left(v_{r}, u_{r}\right)$ and $\left(v_{l}, u_{l}\right)$ are close enough, it is easy to check that $v_{m}>0$ and $u_{m}>0$.

In the case $v_{l}<v_{r}$, the global structure and large time behavior of solutions to the above Riemann problem are studied in (24]). It is shown in (24]) that solutions to the above Riemann problem converge to traveling wave solutions when $v_{l}<v_{r}$. When $v_{l}>v_{r}$, the large time asymptotic behavior will be quite different. We expect that the long time behavior of solutions to the relaxation system (1.2) can be described by that of the "equilibrium" equation (1.4). The large time behavior of solutions for the "equilibrium" equation (1.4) (cf. [20]) is determined by the end states $v_{l}$ and $v_{r}$ as $x \rightarrow \pm \infty$. The long time behavior of solutions to (1.4) is a shock wave when $v_{l}<v_{r}$ and a rarefaction wave when $v_{l}>v_{r}$ (cf. 20]). Therefore, we expect that the solution of the Riemann problem (1.2) and (1.6) will converge to the rarefaction wave of the "equilibrium" equation (1.4) when $v_{l}>v_{r}$ as $t \rightarrow \infty$. One of the purposes in this paper is to confirm this expectation. The proof of the convergence to rarefaction waves in this paper is quite different from that of the convergence to the traveling wave as in 24]. This is because the traveling wave solves (1.2), but the rarefaction wave solution of (1.4) does not solve equation (1.2). This causes much of the difficulty in our analysis.

Here we highlight the new ingredients in this paper, compared with related previous works as follows:

i) A semi-linear model proposed by Jin and Xin (16]) has been extensively studied (cf. [2], [16, 13, 17, 18, [26, 27, 29, 33, 39, 40, 443, 46], 47, 48] and [4]). The feature of the model in [16] is that the left-hand side of the system is linear and the coefficients are constant, while the left-hand side of system (1.2) is nonlinear.

ii) For some $2 \times 2$ hyperbolic systems with relaxation, with the left hand side being nonlinear, the behavior of solutions are studied mainly for smooth solutions. Here we mention two results of this type. First, in [21], convergence to "equilibrium" rarefaction waves and shock profiles is proved respectively for smooth solutions of general $2 \times 2$ 
relaxation systems, with the assumption that the end states at $x= \pm \infty$ are on the "equilibrium" curve. In the present paper, the two end states satisfying (1.7) and (1.8) are not on the "equilibrium" curve $u=f(v)$. For the case when the end states are not on the "equilibrium" curve, the large time behavior of smooth solutions of the $p$-system with relaxation of the form $f(v)-u$ is considered in [54]. The relaxation form is linear in $u$, and $u$ and $v$ are separated, thus simplifying the analysis. For system (1.2), the relaxation form $S-C_{f} u^{2} v$ is more complicated and more physically reasonable, i.e., it is nonlinear in $u$, and $u$ and $v$ are not separated. This causes considerable difficulty in obtaining desired estimates. Therefore, one can expect that the analysis of (1.2) will be more difficult than the $p$-system studied in [54, even for smooth solutions.

iii) There has been some progress in Riemann solutions for some inhomogeneous hyperbolic systems. In [20], the local in time existence and structure of Riemann solutions for general inhomogeneous hyperbolic systems are discussed. In [12] and [11, the global existence of Riemann solutions and convergence to diffusion waves for Riemann solutions containing a single shock for systems with damping are studied, respectively, where the source term is very simple (the right-hand side of the second equation is replaced by $-\alpha u$ ). The source term in (1.2) is much more complicated, and the solutions of the Riemann problem exhibit completely different behavior. In [9, the Riemann problem of a system arising from viscoelasticity is discussed. This system has the form of $u_{t}+\sigma_{x}=0$, $(\sigma-f(u))_{t}+(\sigma-\mu f(u))=0$ with the second equation being an ordinary differential equation for $\sigma-f(u)$ which can be introduced as a new variable $v$ to simplify the arguments. In (24), as mentioned above, convergence to traveling waves for solutions of the Riemann problem of (1.2) and (1.6) is proved for the case $v_{l}<v_{r}$. However, as mentioned above, the proof of convergence to rarefaction waves is quite different from that for traveling waves.

REmark 1. Only the Riemann problem is studied in this paper. The ideas and techniques employed here can also be used to handle the perturbed Riemann problem with the data

$$
(v(x, 0), u(x, 0))=\left\{\begin{array}{lc}
\left(v_{l}(x), u_{l}(x)\right), & -\infty<x<0, \\
\left(v_{r}(x), u_{r}(x)\right) & 0<x<+\infty,
\end{array}\right.
$$

where the functions $\left(v_{l}(x), u_{l}(x)\right)$ and $\left(v_{r}(x), u_{r}(x)\right)$ are small and smooth perturbations of the constant states $\left(v_{l}, u_{l}\right)$ and $\left(v_{r}, u_{r}\right)$. Moreover, a combination of the ideas and techniques used here and those used in 23 . can be used to handle the interaction of shocks.

Relaxation problems have attracted considerable attention in the recent years ([3], 4, [5], 8], 14, 21], 25], 28], 31], 32], 34, 36], 37, 50], [51, 52], 53]). The general setting can be found in 4 . Convergence to planar traveling waves for smooth solutions for the 2-D shallow water wave equation with relaxation is shown in 10. The fluid dynamic limit (cf. [45] and [44) and the boundary layer problem (cf. [19]) of the $3 \times 3$ nonlinear Broadwell model of Boltzmann equations were studied. The Riemann problem of a modified Broadwell model with self-similar structure was investigated in [7] and [38. The existence and stability of shock profiles (traveling waves) for a general nonlinear $n \times n$ system with relaxation were studied in [52] and 31, respectively. For the initial 
boundary value problem, one can refer to [34, 41], 46], 47] and [48. A quasilinear model of gas dynamics equations was investigated in [53. A modified Glimm's scheme ([25]) and a wave front tracking method ([1]) are used respectively to prove the existence of global BV solutions of $p$-system with relaxation for $p(v)=k v^{-1}$.

Closely related to this paper is a result obtained in 15 for system (1.2) with viscosity in the case that the subcharacteristic condition (1.5) is violated, the weakly nonlinear limit is verified, and the underlying relaxation system reduces to the Burgers equation with a source term ( $\mathrm{cf}[15]$ ). It would be important and interesting to study the behavior of discontinuous solutions of the inviscid system (1.2) when the subcharacteristic condition (1.5) is violated. As pointed out in [42, the resulting flow in this case is not necessarily completely chaotic or without structure. In favorable circumstances, it takes the form of "roll waves" with a periodic structure of discontinuous bores separated by smooth profiles.

The rest of the paper is organized as follows. In Section 2, we state the main result of this paper. In Section 3, we gather some decay estimates of the discontinuities, which play an important role in our analysis. In Section 4, some properties of the "equilibrium" rarefaction wave is presented. The problem is reformulated in Section 5. In Section 6, the energy method is used to study the problem formulated in Section 5.

2. Statement of the result. We now present the main results in the paper. Before that, let us define the shock waves for (1.2) as follows.

A discontinuity along $x=x_{1}(t)$ is called a 1-shock if the Rankine- Hugoniot condition

$$
\left\{\begin{array}{l}
\frac{d x_{1}(t)}{d t}=-\sqrt{-\frac{\left.p\left(v_{+}\right)-p_{(} v_{-}\right)}{v_{+}-v_{-}}}, \\
u_{+}-u_{-}=-\dot{x}_{1}(t)\left(v_{+}-v_{-}\right), \\
p\left(v_{+}\right)-p\left(v_{-}\right)=\dot{x}_{1}(t)\left(u_{+}-u_{-}\right),
\end{array}\right.
$$

and the entropy condition

$$
v_{+}(t)<v_{-}(t)
$$

hold, where

$$
\left(v_{-}(t), u_{-}(t)\right)=(v, u)\left(x_{1}(t)-0, t\right),\left(v_{+}(t), u_{+}(t)\right)=(v, u)\left(x_{1}(t)+0, t\right) .
$$

The 2-shock can be defined similarly as a discontinuity $x=x_{2}(t)$ satisfying the RankineHugoniot condition

$$
\left\{\begin{array}{l}
\frac{d x_{2}(t)}{d t}=\sqrt{-\frac{\left.p\left(v_{+}\right)-p_{(} v_{-}\right)}{v_{+}-v_{-}}} \\
u_{+}-u_{-}=-\dot{x}_{2}(t)\left(v_{+}-v_{-}\right), \\
p\left(v_{+}\right)-p\left(v_{-}\right)=\dot{x}_{2}(t)\left(u_{+}-u_{-}\right),
\end{array}\right.
$$

and the entropy condition

$$
v_{+}(t)>v_{-}(t),
$$

where

$$
\left(v_{-}(t), u_{-}(t)\right)=(v, u)\left(x_{2}(t)-0, t\right),\left(v_{+}(t), u_{+}(t)\right)=(v, u)\left(x_{2}(t)+0, t\right) .
$$

The Riemann problem of (1.2) and (1.6) for $0<t \leq T$ can be formulated as the following free boundary problem. 
FBP: 1-shock discontinuity $x=x_{1}(t)$ issuing from $(0,0)$, satisfying the Rankine-Hugoniot condition, the entropy condition

$$
v\left(x_{1}(t)-, t\right)>v\left(x_{1}(t)+, t\right)
$$

and the initial condition

$$
\lim _{t \rightarrow 0}(v, u)\left(x_{1}(t)-, t\right)=\left(v_{l}, u_{l}\right), \lim _{t \rightarrow 0}(v, u)\left(x_{1}(t)+, t\right)=\left(v_{m}, u_{m}\right) ;
$$

while a 2-shock $x=x_{2}(t)$ issuing from $(0,0)$, satisfying the Rankine-Hugoniot condition, the entropy condition

$$
v\left(x_{2}(t)-, t\right)<v\left(x_{2}(t)+, t\right)
$$

and the initial condition

$$
\lim _{t \rightarrow 0}(v, u)\left(x_{2}(t)-, t\right)=\left(v_{m}, u_{m}\right), \quad \lim _{t \rightarrow 0}(v, u)\left(x_{2}(t)+, t\right)=\left(v_{r}, u_{r}\right) .
$$

The solution $(v, u)$ is smooth in the region

$$
S(T)=\left\{(x, t) \mid 0<t \leq T, x_{1}(t) \leq x \leq x_{2}(t)\right\} .
$$

In the outer region $O_{1}(T)=\left\{(x, t) \mid 0 \leq t \leq T,-\infty<x<x_{1}(t)\right\}$, the solution is completely determined by the initial left state $\left(v_{l}, u_{l}\right)$ because of the entropy condition. Similarly, the solution in $O_{2}(T)=\left\{(x, t) \mid 0 \leq t \leq T, x_{2}(t)<x<\infty\right\}$ is completely determined by $\left(v_{r}, u_{r}\right)$. In the following, for simplicity, we set $g^{\prime}=1$. Therefore,

$$
f(v)=\sqrt{\frac{S}{C_{f} v}} .
$$

It is easy to check that the solution in $O_{1}(T)$ is given by

$$
(v, u)(x, t)=\left(v_{l}, u^{l}(x, t)\right)=\left(v_{l}, \sqrt{\frac{S}{C_{f} v_{l}}} \frac{1+y^{l}}{1-y^{l}}\right), x<x_{1}(t),
$$

where

$$
y^{l}=\frac{u_{l} \sqrt{C_{f} v_{l}}-\sqrt{S}}{u_{l} \sqrt{C_{f} v_{l}}+\sqrt{S}} \exp \left(-2 \sqrt{S C_{f} v_{l}} t\right) .
$$

The solution $(v, u)$ in $O_{1}(T)$ can be obtained by solving the following initial value problem of the system of ODEs:

$$
\begin{gathered}
v_{t}=0, u_{t}=\frac{1}{\epsilon}\left(S-C_{f} u^{2} v\right), \\
\left.(v, u)\right|_{t=0}=\left(v_{l}, u_{l}\right) .
\end{gathered}
$$

Similarly, the solution in $O_{2}(T)$ is given by

$$
(v, u)(x, t)=\left(v_{r}, u^{r}(x, t)\right)=\left(v_{r}, \sqrt{\frac{S}{C_{f} v_{l}}} \frac{1+y^{r}}{1-y^{r}}\right), x>x_{2}(t),
$$

where

$$
y^{r}=\frac{u_{r} \sqrt{C_{f} v_{r}}-\sqrt{S}}{u_{r} \sqrt{C_{f} v_{r}}+\sqrt{S}} \exp \left(-2 \sqrt{S C_{f} v_{r}} t\right) .
$$

It follows from (2.3) and (2.5) that

$$
\left|u^{l}(x, t)-f\left(v_{l}\right)\right| \leq O(1)\left|u_{l}-f\left(v_{l}\right)\right| \exp \left(-\sqrt{S C_{f} v_{l}} t\right), x<x_{1}(t)
$$


and

$$
\left|u^{r}(x, t)-f\left(v_{r}\right)\right| \leq O(1)\left|u_{r}-f\left(v_{r}\right)\right| \exp \left(-\sqrt{S C_{f} v_{r}} t\right), x>x_{2}(t) .
$$

Here and in the following, we use $O(1)$ to denote a generic positive bounded quantity independent of $t$. (2.7) and (2.8) indicate that, in the outer region $O_{i}(T), i=1,2$, the solution $(v, u)$ approaches to the equilibrium state $v=f(u)$ exponentially fast.

The local existence of solutions to the above free boundary problem is a simple corollary of Li and Yu's general theorem on quasilinear hyperbolic systems ([20]). In order to extend the local solution for all time, we need to establish a uniform $C^{1}$-estimate in the region $S(T)$ defined above for $T>0$. This will be carried out in Sections 3 and 4 by the observation that the subcharacteristic condition forces the discontinuity of the solution and its derivatives to decay exponentially with respect to time. Thus, as $t \rightarrow \infty$, the solution of the free boundary problem will approach to a continuous function. Note that the large time asymptotic state depends on the relationship between $v_{l}$ and $v_{r}$. As stated in Section 1, when $v_{l}<v_{r}$, the large time asymptotic state of the solution of the Riemann problem (1.2) and (1.6) is a traveling wave, as shown in 24. When $v_{l}>v_{r}$, the Riemann solution of equilibrium equation (1.4) with the Riemann data $\left(v_{l}, v_{r}\right)$ is a rarefaction wave, which is expected to be the asymptotic state of the solution to the Riemann problem (1.2) and (1.6).

The rarefaction wave $V^{R}$ of equilibrium equation (1.4) is the solution to (1.4) with the Riemann initial data

$$
V^{R}(x, 0)= \begin{cases}v_{l}, & -\infty<x<0, \\ v_{r}, & 0<x<+\infty .\end{cases}
$$

The solution $V^{R}$ is given by

$$
V^{R}(x, t)=\left\{\begin{array}{l}
v_{l}, \quad-\infty<x<-f^{\prime}\left(v_{l}\right) t \\
\left(-f^{\prime}\right)^{-1}(x / t), \quad-f^{\prime}\left(v_{l}\right) t \leq x \leq-f^{\prime}\left(v_{r}\right) t, \\
v_{r}, \quad-f^{\prime}\left(v_{r}\right) t<x<+\infty
\end{array}\right.
$$

here and in the following $\left(-f^{\prime}\right)^{-1}$ denotes the inverse of $-f^{\prime}$.

Now we can state our main theorem. In the following $[\ell](x(t))$ denotes the jump of the function $\ell$ along a curve $x=x(t)$, i.e., $[\ell](x(t)=\ell(x(t)+, t)-\ell(x(t)-, t)$.

THEOREM 2.1. [Structure and asymptotic behavior of solutions to the Riemann problem] Suppose $v_{l}>v_{r}$. If $\left|v_{r}-v_{l}\right|+\left|u_{r}-f\left(v_{r}\right)\right|$ is small enough, and the subcharacteristic condition (1.5) holds, then there exists a global smooth solution to the above (FBP) for any $T>0$ in $S(T)$. Moreover, we have the following estimates:

Along the shocks $x=x_{i}(t), i=1,2$,

$$
\left|[u]\left(x_{i}(t)\right)\right|+\left|[v]\left(x_{i}(t)\right)\right|+\left|\left[u_{x}\right]\left(x_{i}(t)\right)\right|+\left|\left[v_{x}\right]\left(x_{i}(t)\right)\right| \leq O(1)\left|v_{r}-v_{l}\right| \exp (-\alpha t),
$$

for some $\alpha>0$. Furthermore,

$$
\lim _{t \rightarrow \infty} \sup _{x_{1}(t) \leq x \leq x_{2}(t)}\left(\left|v(x, t)-V^{R}(x, t)\right|+\left|u(x, t)-f\left(V^{R}\right)(x, t)\right|\right)=0 .
$$


3. Decay estimate of discontinuities. In this section, we gather the decay estimates of the discontinuities of the solutions $(v, u)$ and their derivatives along the shock curves $x=x_{1}(t)$ and $x=x_{2}(t)$, and other estimates. Those estimates are important for the proof of Theorem 2.1 In the following, we always use $w$ and $z$ to denote the Riemann invariants defined in (1.3). The free boundary problem has the boundaries 1-shock $x=x_{1}(t)$ and 2-shock $x=x_{2}(t)$. In the following we denote

$$
S(T)=\left\{(x, t): 0 \leq t \leq T, x_{1}(t) \leq x \leq x_{2}(t)\right\} .
$$

LEMmA 3.1. Let $(v, u)$ be a smooth solution of the free boundary problem (FBP) as stated in section 2 in $S(T)$. Assume the subcharacteristic condition (1.5) is satisfied. Suppose there exist two positive constants $v_{1}$ and $v_{2}$ independent of $t$ such that $v_{1} \leq v(x, t) \leq v_{2}$ as $(x, t) \in S(T)$. Then we have, for some $\gamma>0$,

$$
\left|[u]\left(x_{i}(t)\right)\right|+\left|[v]\left(x_{i}(t)\right)\right|+\left|\left[u_{x}\right]\left(x_{i}(t)\right)\right|+\left|\left[v_{x}\right]\left(x_{i}(t)\right)\right| \leq O(1)\left|v_{r}-v_{l}\right| \exp (-\gamma t),
$$

where [ ] $\left(x_{i}(t)\right)$ denote the usual jump along the curve $x=x_{i}(t)(i=1,2)$

$$
\begin{aligned}
& \left(\left|w_{x}\right|+\left|z_{x}\right|\right)\left(x_{1}(t)+, t\right)+\left(\left|w_{x}\right|+\left|z_{x}\right|\right)\left(x_{2}(t)-, t\right) \\
& \leq O(1)\left(\left|v_{r}-v_{l}\right|+\left|u_{r}-f\left(v_{r}\right)\right|\right) e^{-\gamma t} \\
& \sup _{(x, t) \in S(T)}\left(\left|w_{x}\right|+\left|z_{x}\right|+\left|w_{x x}\right|(x, t)+\left|z_{x x}\right|+|u-f(v)|\right)(x, t) \\
& \leq O(1)\left|v_{r}-v_{l}\right|+\left|u_{r}-f\left(v_{r}\right)\right|,
\end{aligned}
$$

provided $\left|v_{r}-v_{l}\right|+\left|u_{r}-f\left(v_{r}\right)\right|$ is small enough.

The proof of this lemma can be found in [24, where the characteristic method is employed to derive the above estimates. Those estimates are true no matter whether $v_{l}<v_{r}$ or $v_{l}>v_{r}$ (see [24] for details).

4. The equilibrium rarefaction waves. The rarefaction wave $V^{R}$ of equilibrium equation (1.4) given by (2.10) can be approximated as $t \rightarrow \infty$ by the smooth solution $V(x, t)$ of the following Cauchy problem:

$$
\begin{gathered}
V_{t}-f(V)_{x}=0, \\
V(x, 0)=\left(v^{r}+v^{l}\right) / 2+m(x)\left(v^{r}-v^{l}\right) / 2,
\end{gathered}
$$

where $m(x)$ is a smooth function satisfying $m(x)=-1$ for $x \leq-k_{0}, m(x)=1$ for $x \geq k_{0}$ and $m^{\prime}(x) \geq 0$. Here $k_{0}$ is a fixed constant. Since $V_{0}^{\prime}(x) \leq 0$, then $\frac{d\left(-f^{\prime}\left(V_{0}(x)\right)\right.}{d x} \geq 0$, so the solution $V(x, t)$ of (4.1) and (4.2) is an expansion wave and thus smooth. The following lemma gives some estimates on $V$.

Lemma 4.1. Let $V$ be the solution to the Cauchy problem (4.1) and (4.2). Then we have the following estimates:

a)

$$
\lim _{t \rightarrow \infty} \sup _{-\infty<x<+\infty}\left|V-V^{R}\right|(x, t)=0,
$$

where $V^{R}$ is the rarefaction wave $V^{R}$ of equilibrium equation (1.4) given by (2.10). 
b)

$$
V_{x}(x, t) \leq 0
$$

for $x \in \mathbf{R}^{1}$ and $t \geq 0$,

$$
\left|D^{\alpha} V\right|(x, t)=\left\{\begin{array}{l}
0, \quad \text { for }|x| \geq k_{0}+m t, \\
O(1)\left|v_{l}-v_{r}\right|(t+1)^{-|\alpha|},
\end{array} \quad \text { for }|x| \leq k_{0}+m t,\right.
$$

where

$$
m=\max \left\{\left|f^{\prime}(v)\right|: v_{r} \leq v \leq v_{l}\right\}=\left|f^{\prime}\left(v_{r}\right)\right|,
$$

and for any partial derivative $D^{\alpha} V$ of $V$ with respect to $x$ and $t$

$$
V(x, t)= \begin{cases}v_{l}, & \text { for } x<-k_{0}-m t, \\ v_{r}, & \text { for } x>k_{0}+m t .\end{cases}
$$

Proof. a) Any solutions of (4.1) with the same end states are time asymptotically equivalent (i.e., they converge to each other in $L^{\infty}$-norm as $t$ tends to infinity [22]).

b) Since the wave is expansive, i.e. $\frac{\partial\left(-f^{\prime}(V)\right)(x, t)}{\partial x} \geq 0$, and $f^{\prime}(V)<0$ so $V_{x} \leq 0$. This proves (4.4). (4.5) and (4.7) can be found in (21] p. 158).

5. Reformulation of the problem. In order to prove (2.12), it is sufficient to show that

$$
\lim _{t \rightarrow \infty} \sup _{-\infty<x<+\infty}|v-V|(x, t)=0
$$

in view of (4.3). For this purpose, we let

$$
\phi=v-V, \psi=u-f(V) .
$$

Then $\phi$ and $\psi$ satisfy the following system of equations for $x_{1}(t)<x<x_{2}(t)$ :

$$
\begin{gathered}
\phi_{t}-\psi_{x}=0, \\
\psi_{t}+p(V+\phi)_{x}+\left(f^{\prime}(V)\right)^{2} V_{x}=R(\phi, \psi, V),
\end{gathered}
$$

where

$$
R(\phi, \psi, V)=S-C_{f}(\psi+f(V))^{2}(\phi+V) .
$$

We write $p(V+\phi)$ as

$$
p(V+\phi)=P(V)+p^{\prime}(V) \phi+Q(V, \phi) .
$$

Then $Q(V, \phi)$ satisfies

$$
Q(V, \phi)=O(1) \phi^{2}
$$

In this setting, (5.3) can be written as

$$
\psi_{t}+\left(p^{\prime}(V) \phi\right)_{x}=R(\phi, \psi, V)-Q(V, \phi)-F(V)_{x},
$$

where $F(V)$ is a function such that $F^{\prime}(V)=p^{\prime}(V)+\left(f^{\prime}(V)\right)^{2}$, which can be taken as

$$
F(V)=\int_{v_{-}}^{V}\left(p^{\prime}(z)+\left(f^{\prime}(z)\right)^{2}\right) d z
$$

Differentiating (5.7) with respect to $x$, using (5.2), we obtain,

$$
\phi_{t t}+\left(p^{\prime}(V) \phi\right)_{x x}=R_{x}-Q_{x}-F(V)_{x x} .
$$


We will work on equation (5.9) by using the energy method in $S(T)=\{(x, t) \mid 0<t \leq$ $\left.T, x_{1}(t) \leq x \leq x_{2}(t)\right\}$. For this purpose, we establish the following estimates of $\phi$ and its derivatives on the boundaries of $S(T)$.

Lemma 5.1. Suppose there exist two positive constants $v_{1}$ and $v_{2}$ independent of $t$ such that $v_{1} \leq v(x, t) \leq v_{2}$ as $(x, t) \in S(T)$. Then

$$
\begin{gathered}
(|\phi|+|\psi|)\left(x_{1}(t)+, t\right)+(|\phi|+|\psi|)\left(x_{2}(t)-, t\right) \leq O(1)\left(\left|v_{r}-v_{l}\right|+\mid u_{r}-f\left(v_{r}\right)\right) \mid e^{-\gamma t} \\
\quad\left(\left|\phi_{x}\right|+\left|\psi_{x}\right|\right)\left(x_{1}(t)+, t\right)+\left(\left|\phi_{x}\right|+\left|\psi_{x}\right|\right)\left(x_{2}(t)-, t\right) \\
\leq O(1)\left(\left|v_{r}-v_{l}\right|+\left|u_{r}-f\left(v_{r}\right)\right|\right) e^{-\gamma t} \\
\left(\left|D^{2} \phi\right|+\left|D^{2} \psi\right|\right)\left(x_{1}(t)+, t\right)+\left(\left|D^{2} \phi\right|+\left|D^{2} \psi\right|\right)\left(x_{2}(t)-, t\right) \\
\leq O(1)\left(\left|v_{r}-v_{l}\right|+\left|u_{r}-f\left(v_{r}\right)\right|\right)
\end{gathered}
$$

where $\gamma>0$ is a positive constant, provided $\left|v_{r}-v_{l}\right|+\left|u_{r}-f\left(v_{r}\right)\right|$ is small and the subcharacteristic condition (1.5) holds..

Proof. First we note that

$$
\left|\phi\left(x_{1}(t)+, t\right)\right|=|v-V|\left(x_{1}(t)+, t\right) \leq\left|v\left(x_{1}(t)+, t\right)-v_{l}\right|+\left|V\left(x_{1}(t), t\right)-v_{l}\right| .
$$

By (3.2) and the fact $v\left(x_{1}(t)+, t\right)=v_{l}$, we have

$$
\left|v\left(x_{1}(t)+, t\right)-v_{l}\right| \leq O(1)\left(\left|v_{r}-v_{l}\right|+\mid u_{r}-f\left(v_{r}\right)\right) \mid e^{-\gamma t},
$$

for some $\gamma>0$. On the other hand, due to the subcharacteristic condition (1.5), there exists a positive constant $a>0$ such that

$$
\dot{x}_{1}(t)+m<-a,
$$

where $m=-\left|f^{\prime}\left(v_{r}\right)\right|$. So $x_{1}(t)<-m t-a t$ for $t>0$. Therefore, there exists $T_{0}>0$ such that $x_{1}(t)<-k_{0}-m t$ for $t<T_{0}$, where $k_{0}$ is the constant in (5.1). So, by (5.1),

$$
V\left(x_{1}(t), t\right)=v_{l}
$$

for $t>T_{0}$. For $t<T_{0}$, it is obvious that

$$
\left|V\left(x_{1}(t), t\right)-v_{l}\right| \leq\left|v_{r}-v_{l}\right|
$$

due to (4.4). This, together with (5.13) and (5.14), implies

$$
\left|\phi\left(x_{1}(t)+, t\right)\right| \leq O(1)\left(\left|v_{r}-v_{l}\right|+\mid u_{r}-f\left(v_{r}\right)\right) \mid e^{-\gamma t} .
$$

Similarly,

$$
\left|\psi\left(x_{1}(t)+, t\right)\right|=|u-f(V)|\left(x_{1}(t)+, t\right) \leq\left|u\left(x_{1}(t)+, t\right)-f\left(v_{l}\right)\right|+\left|f(V)\left(x_{1}(t), t\right)-f\left(v_{l}\right)\right| .
$$

The term $\left|f(V)\left(x_{1}(t), t\right)-f\left(v_{l}\right)\right|$ can be handled by (5.15) and (5.16).

On the other hand,

$$
\begin{aligned}
& \left|u\left(x_{1}(t)+, t\right)-f\left(v_{l}\right)\right| \\
& \leq\left|u\left(x_{1}(t)+, t\right)-u\left(x_{1}(t)-, t\right)\right|+\left|u\left(x_{1}(t)-, t\right)-f\left(v_{l}\right)\right| \\
& \leq O(1)\left(\left|v_{r}-v_{l}\right|+\mid u_{r}-f\left(v_{r}\right)\right) \mid e^{-\gamma t} .
\end{aligned}
$$


So

$$
\left|\psi\left(x_{1}(t)+, t\right)\right| \leq O(1)\left(\left|v_{r}-v_{l}\right|+\mid u_{r}-f\left(v_{r}\right)\right) \mid e^{-\gamma t} .
$$

Similarly, one can show,

$$
\left|\phi\left(x_{2}(t)-, t\right)\right|+\left|\psi\left(x_{2}(t)-, t\right)\right| \leq O(1)\left(\left|v_{r}-v_{l}\right|+\mid u_{r}-f\left(v_{r}\right)\right) \mid e^{-\gamma t},
$$

and (5.10) follows. Inequality (5.11) can be proved similarly by using (4.5) $)_{1}$ and (3.3), and (5.19) can be proved similarly by using (4.5) 1 and (3.4).

6. Energy estimate. In this section, we prove the following a priori estimate.

Lemma 6.1. Let $t_{0}>0$ be a small time such that the local solution of (1.2) and (1.6) exists in $\left(0, t_{0}\right)$. For any $T>t_{0}$, suppose there exist two positive constants $v_{1}$ and $v_{2}$ independent of $t$ such that $v_{1} \leq v(x, t) \leq v_{2}$ as $(x, t) \in S(T)$. Then we have the following a priori estimate:

$$
\begin{aligned}
& \int_{x_{1}(t)}^{x_{2}(t)} \sum_{j=0}^{3}\left(\left|D^{j} \phi\right|^{2}+\left|D^{j} \psi\right|^{2}\right)(x, t) d x \\
& \quad+\int_{t_{0}}^{t} \int_{x_{1}(s)}^{x_{2}(s)}\left\{\sum_{j=1}^{3}\left(\left|D^{j} \phi\right|^{2}+\left|D^{j} \psi\right|^{2}\right)+\left|V_{x}\right|\left(\phi^{2}+\psi^{2}\right)\right\}(x, s) d x d s \\
& \leq O(1) \int_{x_{1}\left(t_{0}\right)}^{x_{2}\left(t_{0}\right)} \sum_{j=0}^{3}\left(\left|D^{j} \phi\right|^{2}+\left|D^{j} \psi\right|^{2}\right)\left(x, t_{0}\right) d x+O(1)\left(\left|v_{r}-v_{l}\right|+\left|u_{r}-f\left(v_{r}\right)\right|\right),
\end{aligned}
$$

for $t_{0} \leq t \leq T$ provided $\max _{t_{0} \leq t \leq T} \int_{x_{1}(t)}^{x_{2}(t)}\left(\phi^{2}+\psi^{2}\right)(x, t) d x+\delta$ and $\left|v_{r}-v_{l}\right|+\left|u_{r}-f\left(v_{r}\right)\right|$ are small, where $D^{j}$ denote the partial derivatives with respect to $x$ and $t$. Here and in the following,

$$
\delta=\max _{t_{0} \leq t \leq T, x_{1}(t) \leq x \leq x_{2}(t)}(|\phi|+|\psi|)(x, t) .
$$

Theorem 2.1 follows from Lemma 6.1 immediately by the local existence result of [20] and standard continuation argument (cf. 21]).

REMARK 2. If $\left|v_{r}-v_{l}\right|+\left|u_{r}-f\left(v_{r}\right)\right|$ is small, then it follows from (3.4), (3.4) and (4.5) that

$$
\begin{aligned}
& \max _{t_{0} \leq t \leq T, x_{1}(t) \leq x \leq x_{2}(t)}\left(\sum_{j=1}^{2}\left(\left|D^{j} \phi\right|+\left|D^{j} \psi\right|\right)\right) \\
& \leq O(1)\left|v_{r}-v_{l}\right|+\left|u_{r}-f\left(v_{r}\right)\right| .
\end{aligned}
$$

In fact, (3.4) gives the estimates of the derivatives of $x$. By (3.4) and equation (1.2), one can easily get the estimates of the derivatives of $t$. Estimate (6.3) will be used for the energy estimates. 
Proof of Lemma 6.1. Multiplying (5.9) by $\phi$, we have

$$
\left(\phi_{t t}+\left(p^{\prime}(V) \phi\right)_{x x}\right) \phi=\left(R_{x}-Q_{x}-F(V)_{x x}\right) \phi,
$$

for $x_{1}(t)<x<x_{2}(t)$. We estimate each term as follows. For $x_{1}(t)<x<x_{2}(t)$,

$$
\begin{gathered}
\left(\phi_{t t} \phi=\left(\phi_{t} \phi\right)_{t}-\phi_{t}^{2},\right. \\
\left(\left(p^{\prime}(V) \phi\right)_{x x}\right) \phi=\left(\left(p^{\prime}(V) \phi\right)_{x} \phi\right)_{x}-p^{\prime \prime}(V) V_{x} \phi \phi_{x}-p^{\prime}(V) \phi_{x}^{2} .
\end{gathered}
$$

The term $-Q_{x} \phi$ can be estimated as follows. It follows from (5.5) that

$$
Q=\left(\int_{0}^{1} \int_{0}^{\theta} p^{\prime \prime}(V+\beta \phi) d \beta d \theta\right) \phi^{2}=: D(V, \phi) \phi^{2}
$$

so we have

$$
\begin{aligned}
Q_{x} \phi & =D_{x} \phi^{3}+D\left(\phi^{2}\right)_{x} \phi \\
& =D_{x} \phi^{3}+\frac{2}{3} D\left(\phi^{3}\right)_{x} \\
& =D_{x} \phi^{3}+\frac{2}{3}\left(D \phi^{3}\right)_{x}-\frac{2}{3} D_{x} \phi^{3}=\frac{2}{3}\left(D \phi^{3}\right)_{x}+\frac{1}{3} D_{x} \phi^{3} \\
& =\frac{2}{3}\left(D \phi^{3}\right)_{x}+\frac{1}{3}\left(D_{V} V_{x}+D_{\phi} \phi_{x}\right) \phi^{3} .
\end{aligned}
$$

Moreover,

$$
-F_{x x} \phi \leq O(1)\left(V_{x}^{2}|\phi|+\left|V_{x x} \phi\right|\right) .
$$

Therefore, as $x_{1}(t)<x<x_{2}(t)$,

$$
\begin{aligned}
& \left(\phi_{t} \phi\right)_{t}+\left(-p^{\prime}(V) \phi_{x}^{2}\right)-\phi_{t}^{2} \\
& \leq\left(\left(p^{\prime}(V) \phi\right)_{x} \phi-\frac{2}{3} D \phi^{3}\right)_{x}+R_{x} \phi \\
& +O(1)\left(\left|V_{x} \phi \phi_{x}\right|+\left|V_{x} \phi^{3}\right|+\left|\phi^{3} \phi_{x}\right|+V_{x}^{2}|\phi|+\left|V_{x x} \phi\right|\right) .
\end{aligned}
$$

We estimate $R_{x} \phi$ as follows. At first, it is easy to check, since $2 f^{\prime}(V) V+f(V)=0$ and $\phi_{t}=\psi_{x}$, that

$$
\begin{aligned}
& R_{x}=R_{V} V_{x}+R_{\psi} \psi_{x}+R_{\phi} \phi_{t} \\
& \quad=-C_{f}(f(V)+\psi)\left(2 f^{\prime}(V) \phi+\psi\right) V_{x}-2 C_{f}(f(V)+\psi)(\phi+V) \phi_{t} \\
& -C_{f}(f(V)+\psi)^{2} \phi_{x} .
\end{aligned}
$$


After some calculations, we arrive at (in the following formula, $\beta_{i}(i=1,2)$ are quantities satisfying $\left|\beta_{i}\right| \leq O(1) \delta$ with $\delta$ given by (6.2) $)$

$$
\begin{aligned}
R_{x} \phi & \leq S V^{-2} V_{x} \phi^{2}-C_{f} f(V) V_{x} \phi \psi-\frac{1}{2} C_{f}(f(V)+\psi)^{2}\left(\phi^{2}\right)_{x} \\
& -C_{f}(f(V)+\psi)(V+\phi)\left(\phi^{2}\right)_{t}+O(1)\left|V_{x}\right|\left(\phi^{2}+\psi^{2}\right) \\
& =S V^{-2} V_{x} \phi^{2}-C_{f} f(V) V_{x} \phi \psi-\frac{1}{2} C_{f}\left\{(f(V)+\psi)^{2}\left(\phi^{2}\right)\right\}_{x} \\
& -C_{f}\left\{(f(V)+\psi)(V+\phi)\left(\phi^{2}\right)\right\}_{t}+C_{f} \phi^{2}(f(V)+\psi)\left(f^{\prime}(V) V_{x}+\psi_{x}\right) \\
& +C_{f} \phi^{2}\left\{\left(f^{\prime}(V) V_{t}+\psi_{t}\right)(V+\phi)+(f(V)+\psi)\left(V_{t}+\phi_{t}\right)\right\} \\
& +O(1)\left|V_{x}\right|\left(\phi^{2}+\psi^{2}\right) \\
& \leq\left\{S V^{-2}+C_{f} f(V) f^{\prime}(V)\right\} V_{x} \phi^{2}-C_{f} f(V) V_{x} \phi \psi-\frac{1}{2} C_{f}\left\{\left((f(V))^{2}+\beta_{1}\right) \phi^{2}\right\}_{x} \\
& -C_{f}\left\{\left(f(V) V+\beta_{2}\right)\left(\phi^{2}\right)\right\}_{t}+C_{f}(f(V)+\psi) \psi_{x} \phi^{2} \\
& +C_{f}\left(f^{\prime}(V) V+f(V)\right) V_{t} \phi^{2}+C_{f}\left(\phi^{3} \psi_{t}+f(V) \phi^{2} \phi_{t}\right) \\
& +O(1) \delta\left|V_{x}\right|\left(\phi^{2}+\psi^{2}\right) .
\end{aligned}
$$

By virtue of the fact that $f(V)=\sqrt{\frac{S}{C_{f} V}}$ and $V_{t}=f^{\prime}(V) V_{x}$, we obtain

$$
C_{f} f(V) f^{\prime}(V)=-\frac{1}{2} S V^{-2}, C_{f}\left(f^{\prime}(V) V+f(V)\right) V_{t} \phi^{2}=-\frac{1}{4} S V^{-2} V_{x} \phi^{2} .
$$

Also, since $\phi_{t}=\psi_{x}$, we have

$$
\begin{aligned}
& C_{f}\left(\phi^{3} \psi_{t}+f(V) \phi^{2} \phi_{t}\right)+C_{f}(f(V)+\psi) \psi_{x} \phi^{2} \\
& =C_{f} \phi^{3} \psi_{t}+\frac{C_{f}}{3} f(V)\left(\phi^{3}\right)_{t}+\frac{C_{f}}{3}(f(V)+\psi)\left(\phi^{3}\right)_{t} \\
& \leq \frac{2 C_{f}}{3} \phi^{3} \psi_{t}+\frac{2 C_{f}}{3}\left(2 f(V) \phi^{3}+\psi \phi^{3}\right)_{t}+O(1) \delta\left|V_{x}\right| \phi^{2},
\end{aligned}
$$

where $\delta$ is given by (6.2) . (6.12)-(6.14) imply

$$
\begin{aligned}
R_{x} \phi & \leq \frac{1}{2} S V^{-2} V_{x} \phi^{2}-C_{f} f(V) V_{x} \phi \psi+\frac{2 C_{f}}{3} \phi^{3} \psi_{t} \\
& -C_{f}\left\{\left(f(V) V+\beta_{3}\right) \phi^{2}\right\}_{t}-C_{f}\left\{\left(\frac{(f(V))^{2}}{2}+\beta_{4}\right) \phi^{2}\right\}_{x} \\
& +O(1) \delta\left|V_{x}\right|\left(\phi^{2}+\psi^{2}\right),
\end{aligned}
$$

where $\beta_{3}$ and $\beta_{4}$ are quantities satisfying

$$
\left|\beta_{3}\right|+\left|\beta_{4}\right| \leq O(1) \delta
$$

Inequalities (6.10) and (6.15) imply, in view of the fact $\left|V_{x}\right|<0$, that

$$
\begin{aligned}
& A_{t}+\left(-p^{\prime}(V)\right) \phi_{x}^{2}+\frac{1}{2} C_{f} V^{-2}\left|V_{x}\right| \phi^{2}-\phi_{t}^{2} \\
& \leq B_{x}-C_{f} f(V) V_{x} \phi \psi+\frac{2 C_{f}}{3} \phi^{3} \psi_{t} \\
& +O(1)\left(\left|V_{x} \phi \phi_{x}\right|+\phi^{3}\left|\phi_{x}\right|+V_{x}^{2}|\phi|+\left|V_{x x} \phi\right|\right)+O(1) \delta\left|V_{x}\right|\left(\phi^{2}+\psi^{2}\right),
\end{aligned}
$$


as $x_{1}(t) \leq x \leq x_{2}(t)$, where

$$
\begin{gathered}
A=\phi_{t} \phi+\left(C_{f} f(V) V+\beta\right) \phi^{2},|\beta|<O(1) \delta, \\
\left\{\begin{array}{l}
\left.B=\left(p^{\prime}(V) \phi\right)_{x} \phi-\frac{2}{3} D \phi^{3}-C_{f}\left(\frac{(f(V))^{2}}{2}+\beta_{4}\right) \phi^{2}\right), \\
|B| \leq O(1)\left(|\phi|+\left|\phi_{x}\right|\right) .
\end{array}\right.
\end{gathered}
$$

Multiplying (5.9) by $\phi_{t}$, we have

$$
\left(\frac{1}{2} \phi_{t}^{2}\right)_{t}+\left(p^{\prime}(V) \phi\right)_{x x} \phi_{t}=\left(R_{x}-Q_{x}-F(V)_{x x}\right) \phi_{t} .
$$

Each term can be handled as follows:

$$
\left(p^{\prime}(V) \phi\right)_{x x} \phi_{t}=\left(\left(p^{\prime}(V) \phi\right)_{x} \phi_{t}\right)_{x}-\left(p^{\prime}(V) \phi\right)_{x} \phi_{x t} .
$$

On the other hand,

$$
\begin{aligned}
\left(p^{\prime}(V) \phi\right)_{x} \phi_{x t} & =p^{\prime \prime}(V) V_{x} \phi \phi_{x t}+\frac{1}{2} p^{\prime}(V)\left(\phi_{x}^{2}\right)_{t} \\
& =p^{\prime \prime}(V) V_{x} \phi \phi_{x t}+\frac{1}{2}\left(p^{\prime}(V) \phi_{x}^{2}\right)_{t}-\frac{1}{2}\left(p^{\prime \prime}(V) V_{t} \phi_{x}^{2}\right)
\end{aligned}
$$

(6.21) and (6.22) imply

$$
\left(p^{\prime}(V) \phi\right)_{x x} \phi_{t}=\left(\left(p^{\prime}(V) \phi\right)_{x} \phi_{t}\right)_{x}-p^{\prime \prime}(V) V_{x} \phi \phi_{x t}-\frac{1}{2}\left(p^{\prime}(V) \phi_{x}^{2}\right)_{t}+\frac{1}{2} p^{\prime \prime}(V) V_{t} \phi_{x}^{2} .
$$

By (5.5), we have

$$
Q_{x}=\left(p^{\prime}(V+\phi)-p^{\prime}(V)\right)\left(\phi_{x}+V_{x}\right)-p^{\prime \prime}(V) V_{x} \phi .
$$

This implies

$$
\left|Q_{x} \phi_{t}\right| \leq O(1)\left(\left|V_{x}\right||\phi|\left|\phi_{t}\right|+|\phi|\left|\phi_{x}\right|\left|\phi_{t}\right|\right)
$$

Obviously,

$$
\left|F_{x x} \phi_{t}\right| \leq O(1)\left(V_{x}^{2}+\left|V_{x x}\right|\right)\left|\phi_{t}\right| .
$$

So, we have from (6.20), (6.23) -6.26 that

$$
\begin{aligned}
& \left(\frac{1}{2} \phi_{t}^{2}+\frac{1}{2}\left(-p^{\prime}(V)\right) \phi_{x}^{2}\right)_{t}+\frac{1}{2}\left(p^{\prime \prime}(V) V_{t} \phi_{x}^{2}\right) \\
& \leq-\left(\left(p^{\prime}(V) \phi\right)_{x} \phi_{t}\right)_{x}+R_{x} \phi_{t} \\
& +O(1)\left(\left|V_{x} \phi \phi_{x t}\right|+\left|V_{x}\right||\phi|\left|\phi_{t}\right|+|\phi|\left|\phi_{x}\right|\left|\phi_{t}\right|\right)+O(1)\left(V_{x}^{2}+\left|V_{x x}\right|\right)\left|\phi_{t}\right| .
\end{aligned}
$$

We estimate $R_{x} \phi_{t}$ as follows. Since $\phi_{t}=\psi_{x}$, we have

$$
\begin{aligned}
R_{x} \phi_{t} & =\left(R_{\phi} \phi_{x}+R_{\psi} \psi_{x}+R_{V} V_{x}\right) \phi_{t} \\
& =R_{\phi} \phi_{x} \phi_{t}+R_{\psi} \phi_{t}^{2}+R_{V} V_{x} \phi_{t},
\end{aligned}
$$

where $R_{\phi}=\partial_{\phi} R(V, \phi, \psi), R_{V}=\partial_{V} R(V, \phi, \psi)$ and $R_{\psi}=\partial_{\psi} R(V, \phi, \psi)$. Since $R=S-$ $C_{f}(f(V)+\psi)^{2}(V+\phi)$, it is easy to check that

$$
\left|R_{\phi} \phi_{x} \phi_{t}\right| \leq\left|C_{f}(f(V))^{2} \phi_{x} \phi_{t}\right|+O(1)|\psi|\left|\phi_{x}\right|\left|\phi_{t}\right|
$$

and

$$
\left|R_{V} V_{x} \phi_{t}\right| \leq O(1)(|\phi|+|\psi|)\left|V_{x}\right|\left|\phi_{t}\right| .
$$


It follows from (6.28) -6.30$)$ that

$$
R_{x} \phi_{t} \leq R_{\psi} \phi_{t}^{2}+\left|C_{f}(f(V))^{2} \phi_{x} \phi_{t}\right|+O(1)|\psi|\left|\phi_{x}\right|\left|\phi_{t}\right|+O(1)(|\phi|+|\psi|)\left|V_{x}\right|\left|\phi_{t}\right| .
$$

This, together with (6.26), implies

$$
\begin{aligned}
& \frac{1}{2}\left(\phi_{t}^{2}+\left(-p^{\prime}(V)\right) \phi_{x}^{2}\right)_{t}+\left(-R_{\psi}\right) \phi_{t}^{2}+\frac{1}{2} p^{\prime \prime}(V) V_{t} \phi_{x}^{2} \\
& \leq-\left(\left(p^{\prime}(V) \phi\right)_{x} \phi_{t}\right)_{x}+\left|C_{f}(f(V))^{2} \phi_{x} \phi_{t}\right| \\
& +O(1)\left(\left|V_{x} \phi \phi_{x t}\right|+\left|V_{x}\right||\phi|\left|\phi_{t}\right|+|\phi|\left|\phi_{x}\right|\left|\phi_{t}\right|\right)+O(1)\left(V_{x}^{2}+\left|V_{x x}\right|\right)\left|\phi_{t}\right| \\
& +O(1)|\psi|\left|\phi_{x}\right|\left|\phi_{t}\right|+O(1)|\psi|\left|V_{x}\right|\left|\phi_{t}\right| .
\end{aligned}
$$

(6.17) $+k \times(6.32)$, for some positive constant $k$ to be determined later, yields, after some rearrangements,

$$
\begin{aligned}
& \left(A+\frac{k}{2} \phi_{t}^{2}+\frac{k}{2}\left(-p^{\prime}(V)\right) \phi_{x}^{2}\right)_{t}+H_{x}+\left(-p^{\prime}(V)+\frac{k}{2} p^{\prime \prime}(V) V_{t}\right) \phi_{x}^{2} \\
& +\left(\left(-k R_{\psi}\right)-1\right) \phi_{t}^{2}-\left|k C_{f}(f(V))^{2} \phi_{x} \phi_{t}\right|+\frac{1}{2} C_{f} V^{-2}\left|V_{x}\right| \phi^{2} \\
& \leq \text { R.H.S., }
\end{aligned}
$$

where

$$
H=\left(p^{\prime}(V) \phi\right)_{x} \phi_{t}-B,
$$

with $B$ given by (6.19) $)$ R.H.S. is given by

$$
\begin{aligned}
\text { R.H.S. } & =-C_{f} f(V) V_{x} \phi \psi \\
& +\frac{2 C_{f}}{3} \phi^{3} \psi_{t}+O(1)\left(\left|V_{x} \phi \phi_{x t}\right|+\left|V_{x}\right||\phi|\left|\phi_{t}\right|+|\phi|\left|\phi_{x}\right|\left|\phi_{t}\right|\right)+O(1)\left(V_{x}^{2}+\left|V_{x x}\right|\right)\left|\phi_{t}\right| \\
& +O(1)|\psi|\left|\phi_{x}\right|\left|\phi_{t}\right|+O(1)|\psi|\left|V_{x}\right|\left|\phi_{t}\right| \\
& +O(1)\left(\left|V_{x} \phi \phi_{x}\right|+\phi^{3}\left|\phi_{x}\right|+V_{x}^{2}|\phi|+\left|V_{x x} \phi\right|\right)+O(1) \delta\left|V_{x}\right|\left(\phi^{2}+\psi^{2}\right) .
\end{aligned}
$$

We have, from (6.19),

$$
|H| \leq O(1)\left(|\phi|+\left|\phi_{x}\right|+\left|\phi_{t}\right|\right)
$$

and, as given by (6.18),

$$
A=\phi_{t} \phi+\left(C_{f} f(V) V+\beta\right) \phi^{2},|\beta|<O(1) \delta,
$$

and $\delta=\sup (|\phi|+|\psi|)$. We next choose a positive constant $k$ such that

$$
\phi_{t} \phi+C_{f} f(V) V \phi^{2}+\frac{k}{2} \phi_{t}^{2}>0
$$

and

$$
\left(\left(-k R_{\psi}\right)-1\right) \phi_{t}^{2}-\left|k C_{f}(f(V))^{2} \phi_{x} \phi_{t}\right|+\left(-p^{\prime}(V)\right) \phi_{x}^{2}>0 .
$$

At first, since $v_{l}>V>v_{r}$, we have

$$
\phi_{t} \phi+C_{f} f(V) V \phi^{2}+\frac{k}{2} \phi_{t}^{2} \geq C_{f} v_{r} f\left(v_{r}\right) \phi^{2}+\phi_{t} \phi+\frac{k}{2} \phi_{t}^{2} .
$$


Since $k>0$ and $C_{f} v_{r} f\left(v_{r}\right)>0$, in order to guarantee the quadratic form $C_{f} v_{r} f\left(v_{r}\right) \phi^{2}+$ $\phi_{t} \phi+\frac{k}{2} \phi_{t}^{2}$ to be positive definite, we only require $1-2 k C_{f} v_{r} f\left(v_{r}\right)<0$, i.e.,

$$
k>\frac{1}{2 C_{f} v_{r} f\left(v_{r}\right)}=\frac{1}{2 \sqrt{C_{f} S v_{r}}} .
$$

If (6.37) is satisfied, then there exists a positive constant $c_{1}$ such that

$$
\phi_{t} \phi+C_{f} f(V) V \phi^{2}+\frac{k}{2} \phi_{t}^{2} \geq c_{1}\left(\phi^{2}+\phi_{t}^{2}\right) .
$$

On the other hand, from the formula for $R$, we have

$$
-R_{\psi}=2 C_{f}(f(V)+\psi)(\phi+V) \geq 2 C_{f} v_{r} f\left(v_{r}\right)-O(1) \delta,
$$

in view of the fact that $v_{l}>V>v_{r}$. By virtue of (6.39) and the fact that $p(v)=\frac{1}{2} v^{-2}$ and $f(v)=\sqrt{\frac{S}{C_{f} v}}$, we have

$$
\begin{aligned}
& \left(\left(-k R_{\psi}\right)-1\right) \phi_{t}^{2}-\left|k C_{f}(f(V))^{2} \phi_{x} \phi_{t}\right|+\left(-p^{\prime}(V)\right) \phi_{x}^{2} \\
& \geq\left(2 k C_{f} v_{r} f\left(v_{r}\right)-1\right) \phi_{t}^{2}-k S V^{-1}\left|\phi_{x} \phi_{t}\right|+V^{-3} \phi_{x}^{2}-O(1) \delta \phi_{t}^{2} \\
& \geq\left(2 k C_{f} v_{r} f\left(v_{r}\right)-1\right) \phi_{t}^{2}-k S v_{r}^{-1}\left|\phi_{x} \phi_{t}\right|+v_{r}^{-3} \phi_{x}^{2}-O(1) \delta \phi_{t}^{2}-O(1)\left|v_{l}-v_{r}\right|\left(\phi_{x}^{2}+\phi_{t}^{2}\right) .
\end{aligned}
$$

In order to guarantee the quadratic form $\left(2 k C_{f} v_{r} f\left(v_{r}\right)-1\right) \phi_{t}^{2}-k S v_{r}^{-1}\left|\phi_{x} \phi_{t}\right|+v_{r}^{-3} \phi_{x}^{2}$ to be positively definite, we require that

$$
2 k C_{f} v_{r} f\left(v_{r}\right)-1>0
$$

and

$$
\left(k S v_{r}^{-1}\right)^{2}-4\left(2 k C_{f} v_{r} f\left(v_{r}\right)-1\right) v_{r}^{-3}<0,
$$

and note that (6.41) is the same as (6.37). We obtain from (3.51) and the fact $f(v)=$ $\sqrt{\frac{S}{C_{f} v}}$ that

$$
\frac{4 \sqrt{S C_{f}}-2 \sqrt{S\left(4 C_{f}-S\right)}}{S^{2} \sqrt{v_{r}}}<k<\frac{4 \sqrt{S C_{f}}+2 \sqrt{S\left(4 C_{f}-S\right)}}{S^{2} \sqrt{v_{r}}} .
$$

Here the subcharacteristic condition $S<4 C_{f}$ is used. In view of (6.37) and (6.42), we choose $k$ satisfying

$$
\max \left\{\frac{4 \sqrt{S C_{f}}-2 \sqrt{S\left(4 C_{f}-S\right)}}{S^{2} \sqrt{v_{r}}}, \frac{1}{2 \sqrt{C_{f} S v_{r}}}\right\}<k<\frac{4 \sqrt{S C_{f}}+2 \sqrt{S\left(4 C_{f}-S\right)}}{S^{2} \sqrt{v_{r}}} .
$$

Due to the subcharacteristic condition $S<4 C_{f}$, we have $\frac{1}{2 \sqrt{C_{f} S v_{r}}}<\frac{4 \sqrt{S C_{f}}+2 \sqrt{S\left(4 C_{f}-S\right)}}{S^{2} \sqrt{v_{r}}}$, so the positive number satisfying (6.44) can actually be chosen. By this choice of $k$, we have

$$
\left(2 k C_{f} v_{r} f\left(v_{r}\right)-1\right) \phi_{t}^{2}-k S v_{r}^{-1}\left|\phi_{x} \phi_{t}\right|+v_{r}^{-3} \phi_{x}^{2} \geq c_{3}\left(\phi_{t}^{2}+\phi_{x}^{2}\right),
$$

for some positive constant $c_{3}$. This, together with (6.40), implies

$$
\left(\left(-k R_{\psi}\right)-1\right) \phi_{t}^{2}-\left|k C_{f}(f(V))^{2} \phi_{x} \phi_{t}\right|+\left(-p^{\prime}(V)\right) \phi_{x}^{2} \geq c_{4}\left(\phi_{t}^{2}+\phi_{x}^{2}\right),
$$


for some positive constant $c_{4}$, if $\delta=\sup (|\phi|+|\psi|)$ and $\left|v_{l}-v_{r}\right|$ are small. Since $V_{t}=$ $f^{\prime}(V) V_{x}, f^{\prime}(V)<0$ and $V_{x}<0$, we have $V_{t}>0$. So the third term in (6.33) is positive. Moreover, since $f^{\prime}(V)<0$ and $V_{x}<0$, we have $\frac{k}{2} C_{f} f(V) f^{\prime}(V) V_{x} \phi^{2} \geq c_{5}\left|V_{x}\right| \phi^{2}$ for some positive constant $c_{5}$. This, together with (6.46) and (6.33), yields

$$
\begin{aligned}
& \left(A+\frac{k}{2} \phi_{t}^{2}+\frac{k}{2}\left(-p^{\prime}(V)\right) \phi_{x}^{2}\right)_{t}+H_{x}+c_{6}\left(\phi_{t}^{2}+\phi_{x}^{2}\right)+c_{7}\left|V_{x}\right| \phi^{2} \\
& \leq \text { R.H.S., }
\end{aligned}
$$

for some positive constants $c_{6}$ and $c_{7}$, if $\delta=\sup (|\phi|+|\psi|)$ and $\left|v_{l}-v_{r}\right|$ are small, where R.H.S. is given by (6.34). For R.H.S., using Cauchy-Schwarz inequality, we obtain

$$
\begin{aligned}
& \text { R.H.S. } \\
& \leq \frac{1}{2}\left(c_{6}\left(\phi_{t}^{2}+\phi_{x}^{2}\right)+c_{7}\left|V_{x}\right| \phi^{2}\right)+O(1)\left(\left|V_{x}\right| \psi^{2}+\phi^{6}+V_{x}^{4}+V_{x x}^{2}+\delta \phi_{x t}^{2}\right) \\
& +O(1) \delta\left(\phi_{t}^{2}+\phi_{x}^{2}\right)+O(1)\left(V_{x}^{2}+\left|V_{x x}\right|\right)|\phi|+O(1)\left|\phi^{3} \psi_{t}\right| .
\end{aligned}
$$

So, (6.47) and (6.48) imply

$$
\begin{aligned}
& \left(A+\frac{k}{2} \phi_{t}^{2}+\frac{k}{2}\left(-p^{\prime}(V)\right) \phi_{x}^{2}\right)_{t}+H_{x}+\left(\phi_{t}^{2}+\phi_{x}^{2}\right)+\left|V_{x}\right| \phi^{2} \\
& \leq O(1)\left(\left|V_{x}\right| \psi^{2}+\phi^{6}+V_{x}^{4}+V_{x x}^{2}+\delta \phi_{x t}^{2}\right)+O(1)\left(V_{x}^{2}+\left|V_{x x}\right|\right)|\phi| .
\end{aligned}
$$

Integrating (6.49) over the region $\left\{(x, s) \mid x_{1}(s) \leq x \leq x_{2}(s), t_{0} \leq s \leq t\right\}$ and using Green's formula and (6.46), we obtain

$$
\begin{aligned}
& \int_{x_{1}(t)}^{x_{2}(t)} E(x, t) d x+\int_{t_{0}}^{t} \int_{x_{1}(s)}^{x_{2}(s)}\left\{\left(\phi_{t}^{2}+\phi_{x}^{2}\right)+\left|V_{x}\right| \phi^{2}\right\}(x, s) d x d s \\
& \leq \int_{x_{1}\left(t_{0}\right)}^{x_{2}\left(t_{0}\right)} E\left(x, t_{0}\right) d x+O(1) \int_{t_{0}}^{t} \int_{x_{1}(s)}^{x_{2}(s)}\left(\left|V_{x}\right| \psi^{2}+\phi^{6}+V_{x}^{4}+V_{x x}^{2}+\delta \phi_{x t}^{2}+\left|\phi^{3} \psi_{t}\right|\right) d x d s \\
& +O(1) \int_{t_{0}}^{t} \int_{x_{1}(s)}^{x_{2}(s)}\left(V_{x}^{2}+\left|V_{x x}\right|\right)|\phi| d x d s \\
& +\int_{t_{0}}^{t}\left\{E\left(x_{2}(s)-, s\right) \dot{x}_{2}(s)-E\left(x_{1}(s)+, s\right) \dot{x}_{1}(s)+H\left(x_{1}(s)+, s\right)-H\left(x_{2}(s)-, s\right)\right\} d s,
\end{aligned}
$$

where

$$
E=A+\frac{k}{2} \phi_{t}^{2}+\frac{k}{2}\left(-p^{\prime}(V)\right) \phi_{x}^{2}
$$


It follows from (6.38) that

$$
c_{8}\left(\phi^{2}+\phi_{t}^{2}+\phi_{x}^{2}\right) \leq E \leq c_{9}\left(\phi^{2}+\phi_{t}^{2}+\phi_{x}^{2}\right),
$$

for some positive constants $c_{8}$ and $c_{9}$. Moreover, by Lemma 5.1 and (6.35), we have

$$
\begin{aligned}
& \int_{t_{0}}^{t}\left\{E\left(x_{2}(s)-, s\right) \dot{x}_{2}(s)-E\left(x_{1}(s)+, s\right) \dot{x}_{1}(s)+H\left(x_{1}(s)+, s\right)-H\left(x_{2}(s)-, s\right)\right\} d s \\
& \leq O(1)\left(\left|v_{r}-v_{l}\right|+\left|v_{r}-f\left(v_{r}\right)\right|\right) .
\end{aligned}
$$

By Lemma 4.1, we have

$$
\int_{t_{0}}^{t} \int_{x_{1}(s)}^{x_{2}(s)}\left(V_{x}^{4}+V_{x x}^{2}\right) d x d s \leq O(1)\left|v_{r}-v_{l}\right| .
$$

We estimate $\int_{t_{0}}^{t} \int_{x_{1}(s)}^{x_{2}(s)} \phi^{6}(x, s) d x d s$ as follows. At first, for $x_{1}(t)<x<x_{2}(t)$, we have

$$
\phi^{2}(x, t)=\phi^{2}\left(x_{1}(t)-, t\right)+\int_{x_{1}(t)}^{x} 2 \phi \phi_{x}(y, t) d y .
$$

Hence

$$
\begin{aligned}
& \phi^{4}(x, t)=O(1) \phi^{4}\left(x_{1}(t)+, t\right)+\left(\int_{x_{1}(t)}^{x_{2}(t)}\left|\phi \phi_{x}\right|(x, t) d x\right)^{2} \\
& \leq O(1) \phi^{4}\left(x_{1}(t)+, t\right)+\int_{x_{1}(t)}^{x_{2}(t)} \phi^{2}(x, t) d x \int_{x_{1}(t)}^{x_{2}(t)} \phi_{x}^{2}(x, t) d x .
\end{aligned}
$$

Thus, by (5.10) and the fact that $x_{2}(s)-x_{1}(s)=O(1) s$, we have

$$
\begin{aligned}
& \int_{t_{0}}^{t} \int_{x_{1}(s)}^{x_{2}(s)} \phi^{6} d x d s \\
& \leq O(1) \int_{t_{0}}^{t} \phi^{4}\left(x_{1}(s)+, s\right)\left(x_{2}(s)-x_{1}(s)\right) d s \\
& +O(1) \max _{t_{0} \leq s \leq t}\left(\int_{x_{1}(s)}^{x_{2}(s)} \phi^{2}(x, s) d s\right)^{2} \int_{t_{0}}^{t} \int_{x_{1}(s)}^{x_{2}(s)} \phi_{x}^{2} d x d s \\
& \leq O(1)\left(\left|v_{r}-v_{l}\right|+\left|u_{r}-f\left(v_{r}\right)\right|\right)+O(1) \max _{t_{0} \leq s \leq t}\left(\int_{x_{1}(s)}^{x_{2}(s)} \phi^{2}(x, s) d s\right)^{2} \int_{t_{0}}^{t} \int_{x_{1}(s)}^{x_{2}(s)} \phi_{x}^{2} d x d s .
\end{aligned}
$$

Next, we estimate the third term on the right-hand side of (6.50). At first, by (6.55), we have

$$
\max _{x_{1}(t) \leq x \leq x_{2}(t)}|\phi| \leq\left|\phi\left(x_{1}(t)-, t\right)\right|+\left(\int_{x_{1}(t)}^{x_{2}(t)} \phi^{2} d x\right)^{1 / 4}\left(\int_{x_{1}(t)}^{x_{2}(t)} \phi_{x}^{2} d x\right)^{1 / 4} .
$$


Let $I_{3}$ be the third term on the right-hand side of (6.50). It follows from (5.1), (5.10) and (6.57) that

$$
\begin{aligned}
& I_{3} \leq O(1) \int_{t_{0}}^{t}\left(\max _{x_{1}(s) \leq x \leq x_{2}(s)}|\phi|(x, s) \int_{x_{1}(s)}^{x_{2}(s)}\left(V_{x}^{2}+\left|V_{x x}\right|\right) d x\right) d s \\
& \leq O(1) \int_{t_{0}}^{t}\left|\phi\left(x_{1}(s)+, s\right)\right| d s \\
& \left.+\int_{t_{0}}^{t}\left(\int_{x_{1}(s)}^{x_{2}(s)} \phi^{2} d x\right)^{1 / 4}\left(\int_{x_{1}(s)}^{x_{2}(s)} \phi_{x}^{2} d x\right)^{1 / 4} \int_{x_{1}(s)}^{x_{2}(s)}\left(V_{x}^{2}+\left|V_{x x}\right|\right) d x\right) d s \\
& \leq O(1)\left(\left|v_{r}-v_{l}\right|+\left|u_{r}-f\left(v_{r}\right)\right|\right)+\frac{1}{2} \int_{t_{0}}^{t} \int_{x_{1}(s)}^{x_{2}(s)} \phi_{x}^{2} d x d s \\
& +O(1)\left(\max _{t_{0} \leq s \leq t} \int_{x_{1}(s)}^{x_{2}(s)} \phi^{2}(x, s) d x\right)^{1 / 3} \int_{t_{0}}^{t}\left(\int_{x_{1}(s)}^{x_{2}(s)}\left(V_{x}^{2}+\left|V_{x x}\right|\right) d x\right)^{4 / 3} d s \\
& \leq \frac{1}{2} \int_{t_{0}}^{t} \int_{x_{1}(s)}^{x_{2}(s)} \phi_{x}^{2} d x d s+O(1)\left(\left|v_{r}-v_{l}\right|+\left|u_{r}-f\left(v_{r}\right)\right|\right) .
\end{aligned}
$$

So, it follows from (6.50) -6.58$)$ that

$$
\begin{aligned}
& \int_{x_{1}(t)}^{x_{2}(t)}\left(\phi^{2}+\phi_{t}^{2}+\phi_{x}^{2}\right)(x, t) d x+\int_{t_{0}}^{t} \int_{x_{1}(s)}^{x_{2}(s)}\left\{\left(\phi_{t}^{2}+\phi_{x}^{2}\right)+\left|V_{x}\right| \phi^{2}\right\}(x, s) d x d s \\
& \leq O(1) \int_{x_{1}\left(t_{0}\right)}^{x_{2}\left(t_{0}\right)}\left(\phi^{2}+\phi_{t}^{2}+\phi_{x}^{2}\right)\left(x, t_{0}\right) d x+O(1) \int_{t_{0}}^{t} \int_{x_{1}(s)}^{x_{2}(s)}\left(\left|V_{x}\right| \psi^{2}+\delta \phi_{x t}^{2}+\left|\phi^{3} \psi_{t}\right|\right) d x d s \\
& +O(1)\left(\left|v_{r}-v_{l}\right|+\left|u_{r}-f\left(v_{r}\right)\right|\right),
\end{aligned}
$$

if $\left|v_{r}-v_{l}\right|+\left|u_{r}-f\left(v_{r}\right)\right|$ and $\max _{t_{0} \leq s \leq T} \int_{x_{1}(s)}^{x_{2}(s)} \phi^{2}(x, s) d x$ are small. We estimate

$$
\int_{t_{0}}^{t} \int_{x_{1}(s)}^{x_{2}(s)}\left|V_{x}\right| \psi^{2} d x d s
$$

as follows. Multiplying (5.9) by $-V_{x} \psi$, we get

$$
\begin{aligned}
& \left(-\frac{1}{2} V_{x} \psi^{2}\right)_{t}+\frac{1}{2} V_{x t} \psi^{2}-p^{\prime \prime}(V) V_{x}^{2} \phi \psi \\
& =-R V_{x} \psi+Q V_{x} \psi+F(V)_{x} V_{x} \psi
\end{aligned}
$$

Since $C_{f}(f(V))^{2} V=S$, we have

$$
R=-C_{f}\left(2 f(V) \psi+\psi^{2}\right)(\phi+V)
$$

So, we see that

$$
-R V_{x} \phi \leq 2 C_{f} f(V) V V_{x} \psi^{2}+O(1)\left|V_{x}\right| \psi^{2}|\phi| .
$$

From (6.7), we have

$$
\left|Q V_{x} \psi\right| \leq O(1)\left|V_{x}\right||\psi| \phi^{2} .
$$

Obviously, it then follows that

$$
\left|F(V)_{x} V_{x} \psi\right| \leq O(1)\left|V_{x}\right|^{2}|\psi| .
$$


Since $V_{x} \leq 0$, (6.60)-(6.64) and Cauchy-Schwarz inequality imply that

$$
\begin{aligned}
& \left(\frac{1}{2}\left|V_{x} \psi^{2}\right|\right)_{t}+\left|V_{x}\right| \psi^{2} \\
& \leq O(1)\left|v_{r}-v_{l}\right| \phi_{x}^{2}+O(1) \delta\left|V_{x}\right| \phi^{2}+O(1)\left(\left|V_{x}\right|^{2}+\left|V_{x t}\right|\right)|\psi| .
\end{aligned}
$$

Here, we use the fact that $\left|V_{x}\right| \leq\left|v_{r}-v_{l}\right|$ and $\delta=\sup (|\phi|+|\psi|)$. Integrating (6.65) over the region $(x, t): x_{1}(s) \leq x \leq x_{2}(s), t_{0} \leq s \leq t$, and using the (5.10), we obtain

$$
\begin{aligned}
& \int_{x_{1}(t)}^{x_{2}(t)} \frac{1}{2}\left|V_{x} \psi^{2}\right|(x, t) d x+\int_{t_{0}}^{t} \int_{x_{1}(s)}^{x_{2}(s)}\left|V_{x}\right| \psi^{2} d x d s \\
& \leq O(1) \int_{x_{1}\left(t_{0}\right)}^{x_{2}\left(t_{0}\right)} \frac{1}{2}\left|V_{x} \psi^{2}\right|\left(x, t_{0}\right) d x+O(1) \int_{t_{0}}^{t} \int_{x_{1}(s)}^{x_{2}(s)}\left(\left|v_{r}-v_{l}\right| \phi_{x}^{2}+O(1) \delta\left|V_{x}\right| \phi^{2}\right) d x d s \\
& +O(1) \int_{t_{0}}^{t} \int_{x_{1}(s)}^{x_{2}(s)}\left(\left|V_{x}\right|^{2}+\left|V_{x t}\right|\right)|\psi| d x d s+O(1)\left(\left|v_{r}-v_{l}\right|+\left|u_{r}-f\left(v_{r}\right)\right|\right) .
\end{aligned}
$$

Furthermore, (6.59) and (6.66) imply that

$$
\begin{aligned}
& \int_{x_{1}(t)}^{x_{2}(t)}\left(\phi^{2}+\phi_{t}^{2}+\phi_{x}^{2}\right)\left(x, t+\left|V_{x}\right| \psi^{2}\right) d x \\
& +\int_{t_{0}}^{t} \int_{x_{1}(s)}^{x_{2}(s)}\left\{\phi_{t}^{2}+\phi_{x}^{2}+\left|V_{x}\right|\left(\phi^{2}+\psi^{2}\right)\right\}(x, s) d x d s \\
& \leq O(1) \int_{x_{1}\left(t_{0}\right)}^{x_{2}\left(t_{0}\right)}\left(\phi^{2}+\phi_{t}^{2}+\phi_{x}^{2}+\left|V_{x}\right| \psi^{2}\right)\left(x, t_{0}\right) d x+O(1) \int_{t_{0}}^{t} \int_{x_{1}(s)}^{x_{2}(s)}\left(\delta \phi_{x t}^{2}+\left|\phi^{3} \psi_{t}\right|\right) d x d s \\
& +O(1)\left(\left|v_{r}-v_{l}\right|+\left|u_{r}-f\left(v_{r}\right)\right|\right)+O(1) \int_{t_{0}}^{t} \int_{x_{1}(s)}^{x_{2}(s)}\left(\left|V_{x}\right|^{2}+\left|V_{x t}\right|\right)|\psi| d x d s
\end{aligned}
$$

if $\left|v_{r}-v_{l}\right|+\left|u_{r}-f\left(v_{r}\right)\right|$ and $\max _{t_{0} \leq s \leq T} \int_{x_{1}(s)}^{x_{2}(s)} \phi^{2}(x, s) d x$ are small. We use $I_{4}$ to denote the last term in (6.66). Similar to (6.58), we have

$$
\begin{aligned}
& I_{4} \leq \frac{1}{2} \int_{t_{0}}^{t} \int_{x_{1}(s)}^{x_{2}(s)} \psi_{x}^{2} d x d s+O(1)\left(\left|v_{r}-v_{l}\right|+\left|u_{r}-f\left(v_{r}\right)\right|\right) \\
& =\frac{1}{2} \int_{t_{0}}^{t} \int_{x_{1}(s)}^{x_{2}(s)} \phi_{t}^{2} d x d s+O(1)\left(\left|v_{r}-v_{l}\right|+\left|u_{r}-f\left(v_{r}\right)\right|\right), \text { since } \phi_{t}=\psi_{x},
\end{aligned}
$$

if $\max _{t_{0} \leq s \leq T} \int_{x_{1}(s)}^{x_{2}(s)} \psi^{2}(x, s) d x$ is small. So, by (6.67) and (6.68),

$$
\begin{aligned}
& \int_{x_{1}(t)}^{x_{2}(t)}\left(\phi^{2}+\phi_{t}^{2}+\phi_{x}^{2}+\left|V_{x}\right| \psi^{2}\right)(x, t) d x \\
& +\int_{t_{0}}^{t} \int_{x_{1}(s)}^{x_{2}(s)}\left\{\phi_{t}^{2}+\phi_{x}^{2}+\left|V_{x}\right|\left(\phi^{2}+\psi^{2}\right)\right\}(x, s) d x d s \\
& \leq O(1) \int_{x_{1}\left(t_{0}\right)}^{x_{2}\left(t_{0}\right)}\left(\phi^{2}+\phi_{t}^{2}+\phi_{x}^{2}+\left|V_{x}\right| \psi^{2}\right)\left(x, t_{0}\right) d x+O(1) \int_{t_{0}}^{t} \int_{x_{1}(s)}^{x_{2}(s)}\left(\delta \phi_{x t}^{2}+\left|\phi^{3} \psi_{t}\right|\right) d x d s \\
& +O(1)\left(\left|v_{r}-v_{l}\right|+\left|u_{r}-f\left(v_{r}\right)\right|\right),
\end{aligned}
$$


if $\left|v_{r}-v_{l}\right|+\left|u_{r}-f\left(v_{r}\right)\right|$ and $\max _{t_{0} \leq s \leq T} \int_{x_{1}(s)}^{x_{2}(s)}\left(\phi^{2}+\psi^{2}\right)(x, s) d x+\delta$ are small. We turn to the estimate of $\int_{t_{0}}^{t} \int_{x_{1}(s)}^{x_{2}(s)}\left|\phi^{3} \psi_{t}\right| d x d s$. Differentiate (5.7) with respect to $t$ and multiply by $\psi_{t}$ to get

$$
\frac{1}{2}\left(\psi_{t}^{2}\right)_{t}=R_{t} \psi_{t}-\left(\left(p^{\prime}(V) \phi\right)_{x t}+Q_{t}+F(V)_{x t}\right) \psi_{t} .
$$

Similar to (6.11), we have

$$
\begin{aligned}
& R_{t}=-C_{f}(f(V)+\psi)\left(2 f^{\prime}(V) \phi+\psi\right) V_{t}-2 C_{f}(f(V)+\psi)(\phi+V) \psi_{t} \\
& \quad-C_{f}(f(V)+\psi)^{2} \phi_{t} .
\end{aligned}
$$

Since $V>v_{r}$, thus $f(V) V>f\left(v_{r}\right) v_{r}>0$. Moreover, $\left|V_{t}\right|=O(1)\left|V_{x}\right|$. Therefore, (6.71) implies

$$
R_{t} \psi_{t} \leq-2 C_{f} f\left(v_{r}\right) v_{r} \psi_{t}^{2}+O(1)\left(\delta \psi_{t}^{2}+\left|\phi_{t}\right|\left|\psi_{t}\right|\right)+O(1)\left|V_{x}\right|(|\phi|+|\psi|)\left|\psi_{t}\right| .
$$

Moreover, it is easy to verify, with the help of (4.1), (5.8), (6.7) and the Cauchy-Schwarz inequality, that

$$
\begin{aligned}
& \left|\left(\left(p^{\prime}(V) \phi\right)_{x t}+Q_{t}+F(V)_{x t}\right) \psi_{t}\right| \\
& \leq O(1)\left(V_{x}^{4}+V_{x x}^{2}\right)+O(1)\left(\phi_{t}^{2}+\phi_{x t}^{2}\right)+C_{f} f\left(v_{r}\right) v_{r} \psi_{t}^{2},
\end{aligned}
$$

if $|\phi|+|\psi|$ is small. Therefore, (6.70), (6.72) and (6.73) imply, by using the CauchySchwarz inequality,

$$
\frac{1}{2}\left(\psi_{t}^{2}\right)_{t}+\psi_{t}^{2} \leq O(1)\left(V_{x}^{4}+V_{x x}^{2}+\phi_{t}^{2}+\phi_{x t}^{2}\right)+O(1)\left|V_{x}\right|\left(|\phi|^{2}+|\psi|^{2}\right),
$$

if $\delta$ is small. Integrating (6.74) over the region $\left\{(x, s) \mid x_{1}(s) \leq x \leq x_{2}(s), t_{0} \leq s \leq t\right\}$, using (6.54), we obtain

$$
\begin{aligned}
& \int_{x_{1}(t)}^{x_{2}(t)} \psi_{t}^{2}(x, t) d x+\int_{t_{0}}^{t} \int_{x_{1}(s)}^{x_{2}(s)} \psi_{t}^{2}(x, s) d x d s \\
& \leq O(1) \int_{x_{1}\left(t_{0}\right)}^{x_{2}\left(t_{0}\right)}\left(\psi_{t}^{2}\right)\left(x, t_{0}\right) d x+O(1) \int_{t_{0}}^{t} \int_{x_{1}(s)}^{x_{2}(s)}\left(\phi_{t}^{2}+\phi_{x t}^{2}\right) d x d s \\
& +\int_{t_{0}}^{t} \int_{x_{1}(s)}^{x_{2}(s)}\left|V_{x}\right|\left(|\phi|^{2}+|\psi|^{2}\right) d x d s+O(1)\left(\left|v_{r}-v_{l}\right|\right) .
\end{aligned}
$$

For the term $\int_{t_{0}}^{t} \int_{x_{1}(s)}^{x_{2}(s)}\left|\phi^{3} \psi_{t}\right| d x d s$ in (6.69), we can use the Cauchy-Schwarz inequality to obtain

$$
\int_{t_{0}}^{t} \int_{x_{1}(s)}^{x_{2}(s)}\left|\phi^{3} \psi_{t}\right| d x d s \leq O(1) \frac{1}{\epsilon} \int_{t_{0}}^{t} \int_{x_{1}(s)}^{x_{2}(s)} \phi^{6} d x d s+\epsilon \int_{t_{0}}^{t} \int_{x_{1}(s)}^{x_{2}(s)} \psi_{t}^{2},
$$

for any $\epsilon>0 . \int_{t_{0}}^{t} \int_{x_{1}(s)}^{x_{2}(s)} \phi^{6} d x d s$ can be estimated by (6.56).

Next, we turn to the higher order estimates. This is similar to the above estimates and the method used in 21], the difference being that we will need the boundary estimates (5.10) -(5.12). So we only sketch the proof as follows. 
Here and in the following, we set

$$
\delta_{2}=\max _{t_{0} \leq t \leq T, x_{1}(t) \leq x \leq x_{2}(t)}\left(\sum_{j=1}^{2}\left(\left|D^{j} \phi\right|+\left|D^{j} \psi\right|\right)\right),
$$

and by (6.3), we have

$$
\delta_{2} \leq O(1)\left(\left|v_{r}-v_{l}\right|+\left|u_{r}-f\left(v_{r}\right)\right|\right) .
$$

At first, we differentiate (5.9) with respect to $t$ and multiply by $\phi_{t x}$. Then we integrate the resulting equation to obtain

$$
\begin{aligned}
& \int_{x_{1}(t)}^{x_{2}(t)} \phi_{x t}^{2}(x, t) d x+\int_{t_{0}}^{t} \int_{x_{1}(s)}^{x_{2}(s)} \phi_{x t}^{2}(x, s) d x d s \\
& \leq O(1) \int_{x_{1}\left(t_{0}\right)}^{x_{2}\left(t_{0}\right)}\left(\phi_{x t}^{2}+\phi_{x x}^{2}\right)\left(x, t_{0}\right) d x \\
& +O(1) \int_{t_{0}}^{t} \int_{x_{1}(s)}^{x_{2}(s)} \delta_{2}\left(\phi_{x x t}^{2}+\phi_{x t t}^{2}+|\nabla \phi|^{2}+|\nabla \psi|^{2}+\left|D^{2}(\phi, \psi)\right|^{2}+\left|V_{x}\right|\left(\phi^{2}+\psi^{2}\right)\right) d x d s \\
& +O(1)\left(\left|v_{r}-v_{l}\right|+\left|u_{r}-f\left(v_{r}\right)\right|\right) .
\end{aligned}
$$

Therefore, by (6.56), (6.69), (6.76) and (6.79), we can choose $\epsilon$ suitably in (6.76) such that

$$
\begin{aligned}
& \int_{x_{1}(t)}^{x_{2}(t)}\left(\phi^{2}+\phi_{t}^{2}+\phi_{x}^{2}\right)+\left|V_{x}\right| \psi^{2}+\phi_{x t}^{2}(x, t) d x \\
& +\int_{t_{0}}^{t} \int_{x_{1}(s)}^{x_{2}(s)}\left\{\phi_{t}^{2}+\phi_{x}^{2}+\left|V_{x}\right|\left(\phi^{2}+\psi^{2}\right)+\phi_{x t}^{2}\right\}(x, s) d x d s \\
& \leq O(1) \int_{x_{1}\left(t_{0}\right)}^{x_{2}\left(t_{0}\right)}\left(\phi^{2}+\phi_{t}^{2}+\phi_{x}^{2}+\left|V_{x}\right| \psi^{2}+\psi_{t}^{2}+\phi_{x t}^{2}+\phi_{x x}^{2}\right)\left(x, t_{0}\right) d x \\
& +O(1) \int_{t_{0}}^{t} \int_{x_{1}(s)}^{x_{2}(s)} \delta_{2}\left(\phi_{x x t}^{2}+\phi_{x t t}^{2}+\left|D^{2}(\phi, \psi)\right|^{2}\right) d x d s \\
& +O(1)\left(\left|v_{r}-v_{l}\right|+\left|u_{r}-f\left(v_{r}\right)\right|\right),
\end{aligned}
$$

if $\left|v_{r}-v_{l}\right|+\left|u_{r}-f\left(v_{r}\right)\right|$ and $\max _{t_{0} \leq s \leq T} \int_{x_{1}(s)}^{x_{2}(s)}\left(\phi^{2}+\psi^{2}\right)(x, s) d x+\delta$ are small. We differentiate (5.9) with respect to $x$ and multiply by $\phi_{x}$, differentiate (5.9) with respect to $t$ and multiply by $\phi_{t t}$, and differentiate (5.9) with respect to $x$ twice and multiply by respectively $\phi_{x x}$ and $\phi_{x x t}$. We integrate these resulting equations respectively to get

$$
\begin{aligned}
& \int_{x_{1}(t)}^{x_{2}(t)} \phi_{x}^{2}(x, t) d x+\int_{t_{0}}^{t} \int_{x_{1}(s)}^{x_{2}(s)} \phi_{x x}^{2}(x, s) d x d s \\
& \leq O(1) \int_{x_{1}\left(t_{0}\right)}^{x_{2}\left(t_{0}\right)} \phi_{x}^{2}\left(x, t_{0}\right) d x \\
& +O(1) \int_{t_{0}}^{t} \int_{x_{1}(s)}^{x_{2}(s)}\left(\phi_{x t}^{2}+\delta_{2}\left(|\nabla \phi|^{2}+|\nabla \psi|^{2}\right)+\left|V_{x}\right|\left(\phi^{2}+\psi^{2}\right)+\left|V_{x}\right|^{3}\right) d x d s \\
& +O(1)\left(\left|v_{r}-v_{l}\right|+\left|u_{r}-f\left(v_{r}\right)\right|\right),
\end{aligned}
$$




$$
\begin{aligned}
& \int_{x_{1}(t)}^{x_{2}(t)} \phi_{t t}^{2}(x, t) d x+\int_{t_{0}}^{t} \int_{x_{1}(s)}^{x_{2}(s)} \phi_{t t}^{2}(x, s) d x d s \\
& \leq O(1) \int_{x_{1}\left(t_{0}\right)}^{x_{2}\left(t_{0}\right)}\left(\phi_{t t}^{2}+\phi_{x t}^{2}\right)\left(x, t_{0}\right) d x+O(1) \int_{x_{1}(t)}^{x_{2}(t)} \phi_{x t}^{2}(x, t) d x \\
& +O(1) \int_{t_{0}}^{t} \int_{x_{1}(s)}^{x_{2}(s)} \delta_{2}\left(\phi_{x t t}^{2}+\phi_{t t t}^{2}\right)+|\nabla \phi|^{2}+|\nabla \psi|^{2}+\left|D^{2}(\phi, \psi)\right|^{2}+\left|V_{x}\right|\left(\phi^{2}+\psi^{2}\right) d x d s \\
& +O(1)\left(\left|v_{r}-v_{l}\right|+\left|u_{r}-f\left(v_{r}\right)\right|\right), \\
& \int_{x_{1}(t)}^{x_{2}(t)} \phi_{x x}^{2}(x, t) d x+\int_{t_{0}}^{t} \int_{x_{1}(s)}^{x_{2}(s)} \phi_{x x x}^{2}(x, s) d x d s \\
& \leq O(1) \int_{x_{1}\left(t_{0}\right)}^{x_{2}\left(t_{0}\right)}\left(\phi_{x x}^{2}+\phi_{x x t}^{2}\right)\left(x, t_{0}\right) d x+O(1) \int_{x_{1}(t)}^{x_{2}(t)} \phi_{x x t}^{2}(x, t) d x \\
& +O(1) \int_{t_{0}}^{t} \int_{x_{1}(s)}^{x_{2}(s)} \delta_{2}\left(\psi_{x x x}^{2}+|\nabla \phi|^{2}+|\nabla \psi|^{2}+\left|D^{2}(\phi, \psi)\right|^{2}+\left|V_{x}\right|\left(\phi^{2}+\psi^{2}\right)+\left|V_{x}\right|^{3}\right) d x d s \\
& +O(1)\left(\left|v_{r}-v_{l}\right|+\left|u_{r}-f\left(v_{r}\right)\right|\right) .
\end{aligned}
$$

Differentiating (5.9) with respect to $t$ and then with respect to $x$, multiplying by $\phi_{t t x}$, and integrating the resulting equation, we obtain

$$
\begin{aligned}
& \int_{x_{1}(t)}^{x_{2}(t)}\left(\phi_{x x x}^{2}+\phi_{x x t}^{2}\right)(x, t) d x+\int_{t_{0}}^{t} \int_{x_{1}(s)}^{x_{2}(s)} \phi_{x x t}^{2}(x, s) d x d s \\
& \leq O(1) \int_{x_{1}\left(t_{0}\right)}^{x_{2}\left(t_{0}\right)} \phi_{x x x}^{2}\left(x, t_{0}\right) d x+O(1) \int_{x_{1}(t)}^{x_{2}(t)} \phi_{x x t}^{2}(x, t) d x \\
& +O(1) \int_{t_{0}}^{t} \int_{x_{1}(s)}^{x_{2}(s)} \delta_{2}\left(|\nabla \phi|^{2}+|\nabla \psi|^{2}+\left|D^{2}(\phi, \psi)\right|^{2}+\left|D^{3}(\phi, \psi)\right|^{2}\right. \\
& \left.+\left|V_{x}\right|\left(\phi^{2}+\psi^{2}\right)+\left|V_{x}\right|^{3}\right) d x d s \\
& +O(1)\left(\left|v_{r}-v_{l}\right|+\left|u_{r}-f\left(v_{r}\right)\right|\right) .
\end{aligned}
$$

Differentiating (5.9) with respect to $t$ twice, multiplying by $\phi_{t t t}$, and integrating the resulting equation, we get

$$
\begin{aligned}
& \int_{x_{1}(t)}^{x_{2}(t)}\left(\phi_{x t t}^{2}+\phi_{x x t}^{2}\right)(x, t) d x+\int_{t_{0}}^{t} \int_{x_{1}(s)}^{x_{2}(s)} \phi_{x t t}^{2}(x, s) d x d s \\
& \leq O(1) \int_{x_{1}\left(t_{0}\right)}^{x_{2}\left(t_{0}\right)} \phi_{x t t}^{2}\left(x, t_{0}\right) d x+O(1) \int_{x_{1}(t)}^{x_{2}(t)} \phi_{x x t}^{2}(x, t) d x \\
& +O(1) \int_{t_{0}}^{t} \int_{x_{1}(s)}^{x_{2}(s)} \delta_{2}\left(|\nabla \phi|^{2}+|\nabla \psi|^{2}+\left|D^{2}(\phi, \psi)\right|^{2}+\left|D^{3}(\phi, \psi)\right|^{2}\right. \\
& \left.+\left|V_{x}\right|\left(\phi^{2}+\psi^{2}\right)+\left|V_{x}\right|^{3}\right) d x d s \\
& +O(1)\left(\left|v_{r}-v_{l}\right|+\left|u_{r}-f\left(v_{r}\right)\right|\right) .
\end{aligned}
$$


When we get these two estimates, the boundary terms are bounded by

$$
O(1) \int_{t_{0}}^{t}\left(\sum_{j=0}^{1}\left|D^{j}(\phi, \psi)\left\|D^{2}(\phi, \psi)\left(x_{1}(s)+, s\right)+\sum_{j=0}^{1} \mid D^{j}(\phi, \psi)\right\| D^{2}(\phi, \psi)\left(x_{2}(s)-, s\right)\right) d s .\right.
$$

By (5.10) -(5.12), this is bounded by $O(1)\left(\left|v_{r}-v_{l}\right|+\left|u_{r}-f\left(v_{r}\right)\right|\right)$. Following a similar argument as in 21], using Lemma 5.1, one can get the estimate on $\psi$,

$$
\begin{aligned}
& \int_{x_{1}(t)}^{x_{2}(t)} \sum_{j=0}^{3}\left|D^{j} \psi\right|^{2}(x, t) d x+\int_{t_{0}}^{t} \int_{x_{1}(s)}^{x_{2}(s)} \sum_{j=0}^{3}\left|D^{j} \psi\right|^{2}(x, s) d x d s \\
& \leq O(1) \int_{x_{1}\left(t_{0}\right)}^{x_{2}\left(t_{0}\right)} \sum_{j=0}^{3}\left|D^{j} \psi\right|^{2}\left(x, t_{0}\right) d x+O(1) \int_{x_{1}(t)}^{x_{2}(t)} \sum_{j=1}^{3}\left|D^{j} \phi\right|^{2}(x, t) d x \\
& +O(1) \int_{t_{0}}^{t} \int_{x_{1}(s)}^{x_{2}(s)}\left(\sum_{j=1}^{3}\left|D^{j} \phi\right|^{2}+\left|V_{x}\right| \phi^{2}\right) d x d s \\
& +O(1)\left(\left|v_{r}-v_{l}\right|+\left|u_{r}-f\left(v_{r}\right)\right|\right) .
\end{aligned}
$$

Again, using a similar argument as in [21, and (6.80)-(6.86), Lemma 6.1 follows. Theorem 2.1 follows from Lemma 6.1 immediately by the local existence result of [20] and standard continuation argument (cf. [21]).

7. Acknowledgment. The authors are grateful to the referee for his very helpful suggestions and to Professor Wen-Qing Xu for reading through the manuscript. Luo is partially supported by a startup fund of Georgetown University.

\section{REFERENCES}

[1] Amadori, D.; Guerra, G., Global BV solutions and relaxation limit for a system of conservation laws. Proc. Roy. Soc. Edinburgh Sect. A 131 (2001), no. 1, 1-26. MR1820292 (2002a:35135)

[2] Bianchini, S., A Glimm type functional for a special Jin-Xin relaxation model. Ann. Inst. H. Poincaré Anal. Non Linéaire 18 (2001), no. 1, 19-42. MR.1810269 (2002m:49019)

[3] Chern, I. L., Long-time effect of relaxation for hyperbolic conservation laws. Comm. Math. Phys. 172 (1995), no. 1, 39-55. MR1346371 (96j:35151)

[4] Chen, G.-Q.; Levermore, C.D.; Liu, P.-T., Hyperbolic conservation laws with stiff relaxation terms and entropy, Comm. Pure Appl. Math., XLVII (1994), 1-45. MR1280989 (95h:35133)

[5] Chen, G. Q.; Liu, T. P., Zero relaxation and dissipation limits for hyperbolic conservation laws. Comm. Pure Appl. Math. 46 (1993), no. 5, 755-781. MR1213992 (94b:35167)

[6] Courant, R; Friedrichs K. O., Supersonic Flow and Shock Waves, Interscience Publishers Inc., New York, 1948. MR0029615 (10:637c)

[7] Fan H., Self-similar solutions for a modified Broadwell model and its hydrodynamic limits, SIAM J. Math. Anal. 28 (1997), 831-851. MR1453308 (98i:82053)

[8] Fan, H.; Jin, S.; Miller, J., Wave patterns, stability, and slow motions in inviscid and viscous hyperbolic equations with stiff reaction terms. J. Differential Equations 189 (2003), no. 1, 267-291. MR $1968322(2004 c: 35257)$

[9] Greenberg, J; Hsiao L., The Riemann problem for $u_{t}+\sigma_{x}=0$ and $(\sigma-f(u))_{t}+(\sigma-\mu f(u))=0$. Arch. Ration. Mech. Anal. 82 (1983), 87-108. MR0684415 (84k:35126)

[10] Ha, S.; Yu, S. H., Wave front tracing and asymptotic stability of planar traveling waves for a twodimensional shallow river model. J. Differential Equations 186 (2002), no. 1, 230-258. MR1941099 (2003j:76017) 
[11] Hsiao L.; Luo, T., Nonlinear diffusive phenomena of entropy weak solutions for a system of quasilinear hyperbolic conservation laws with damping. Quart. Appl. Math. 56 (1998), no. 1, 173-189. MR:1604829 (98m:35129)

[12] Hsiao, L.; Tang, S. Q., Construction and qualitative behavior of the solution of the perturbated Riemann problem for the system of one-dimensional isentropic flow with damping. J. Differential Equations 123 (1995), no. 2, 480-503. MR1362883 (97d:35137)

[13] Hsiao, L.; Li, H.L.; Mei, M., Convergence rates to superposition of two travelling waves of the solutions to a relaxation hyperbolic system with boundary effects, Math. Models Methods Appl. Sci. 11 (2001), no. 7, 1143-1168. MR.1848195 (2003f:35191)

[14] Hsiao, L.; Pan, R., Nonlinear stability of rarefaction waves for a rate-type viscoelastic system. Chinese Ann. Math. Ser. B 20 (1999), no. 2, 223-232. MR1699147 (2000e:35131)

[15] Jin, S.; Katsoulakis, M., Hyperbolic systems with supercharacteristic relaxations and roll waves, SIAM J. Appl. Math. 61 (2000), no. 1, 273-292 (electronic). MR1776396 (2001h:35116)

[16] Jin, S.; Xin, Z., The relaxation schemes for systems of conservation laws in arbitrary space dimensions. Comm. Pure Appl. Math. 48 (1995), no. 3, 235-276. MR1322811(96c:65134)

[17] Liu, H. L., The $L^{p}$ stability of relaxation rarefaction profiles. J. Differential Equations 171 (2001), no. 2, 397-411. MR1818655(2002a:35132)

[18] Liu, H. L., Asymptotic stability of relaxation shock profiles for hyperbolic conservation laws. J. Differential Equations 192 (2003), no. 2, 285-307. MR.1990842 (2004d:35165)

[19] Liu, J.-G.; Xin, Z., Boundary-layer behavior in the fluid-dynamic limit for a nonlinear model Boltzmann equation. Arch. Rational Mech. Anal. 135 (1996), no. 1, 61-105. MR1414294 (98j:76127)

[20] Li, T. T.; Yu W.C., Boundary value problems for quasilinear hyperbolic systems. Duke University Mathematics Series, V. Duke University, Mathematics Department, Durham, NC, 1985. MR0823237 (88g:35115)

[21] Liu, T.-P., Hyperbolic conservation laws with relaxation, Comm. Math. Phys. 108 (1987), 153-175. MR0872145 (88f:35092)

[22] Liu, T. P., Linear and nonlinear large time behavior of general systems of hyperbolic conservation laws, Comm. Pure Appl. Math. 30 (1977), 767-796. MR0499781(58:17556)

[23] Luo, T.; Yang, T., Interaction of elementary waves for the compressible Euler equations with frictional damping, J. Differential Equations 161 (2000), no. 1, 42-86. MR1740357 (2001c:35182)

[24] Luo, T.; Yang, T., Global Structure and Asymptotic Behavior of Weak Solutions to Flood Wave Equations, J. Differential Equations 207 (2004), no. 1, 117-160. MR2100816

[25] Luo, T.; Natalini, R.; Yang, T., Global BV solutions to a $p$-system with relaxation. J. Differential Equations 162 (2000), no. 1, 174-198. MR1741876 (2001d:35124)

[26] Luo, T.; Xin, Z., Nonlinear stability of shock fronts for a relaxation system in several space dimensions. J. Differential Equations 139 (1997), no. 2, 365-408. MR1472353 (99c:35155)

[27] Luo, T., Asymptotic stability of planar rarefaction waves for the relaxation approximation of conservation laws in several dimensions. J. Differential Equations 133 (1997), no. 2, 255-279. MR.1427853 (98d:35140)

[28] Marcati, P.; Rubino, B., Hyperbolic to parabolic relaxation theory for quasilinear first order systems. J. Differential Equations 162 (2000), no. 2, 359-399. MR.1751710 (2001d:35125)

[29] Mascia, C.; Natalini, R., $L^{1}$ nonlinear stability of traveling waves for a hyperbolic system with relaxation. J. Differential Equations 132 (1996), no. 2, 275-292. MR1422120 (98d:35134)

[30] Mascia, C.; Sinestrari, C.; The perturbed Riemann problem for a balance law. Adv. Differential Equations 2 (1997), no. 5, 779-810. MR1751427(2001e:35115)

[31] Mascia, C.; Zumbrun, K., Pointwise Green's function bounds and stability of relaxation shocks. Indiana Univ. Math. J. 51 (2002), no. 4, 773-904. MR1947862 (2003k:35151)

[32] Natalini, R., Recent results on hyperbolic relaxation problems. Analysis of systems of conservation laws (Aachen, 1997), 128-198, Chapman \& Hall/CRC Monogr. Surv. Pure Appl. Math., 99, Chapman \& Hall/CRC, Boca Raton, FL, 1999. MR.1679940 (2000a:35157)

[33] Natalini, R., Convergence to equilibrium for the relaxation approximations of conservation laws. Comm. Pure Appl. Math. 49 (1996), no. 8, 795-823. MR1391756 (97c:35131)

[34] Nishibata, S; Yu, S. H., The asymptotic behavior of the hyperbolic conservation laws with relaxation on the quarter-plane. SIAM J. Math. Anal. 28 (1997), no. 2, 304-321. MR1434037 (98h:35154)

[35] Smoller, J., Shock waves and reaction-diffusion equations, Springer-Verlag, New York, 1996.

[36] Shen, W.; Tveito, A.; Winther, R., On the zero relaxation limit for a system modeling the motions of a viscoelastic solid. SIAM J. Math. Anal. 30 (1999), no. 5, 1115-1135. MR1709789(2000f:74032) 
[37] Serre, D., The stability of constant equilibrium states in relaxation models. Ann. Univ. Ferrara Sez. VII (N.S.) 48 (2002), 253-274. MR.1980835(2004b:35214)

[38] Slemrod, M.; Tzavaras, A. E., Self-similar fluid-dynamic limits for the Broadwell system. Arch. Rational Mech. Anal. 122 (1993), no. 4, 353-392. MR1217593 (94c:82072)

[39] Tadmor, E.; Tang, T., Pointwise error estimates for relaxation approximations to conservation laws. SIAM J. Math. Anal. 32 (2000), no. 4, 870-886. MR1814742 (2001m:65121)

[40] Teng, Z.-H., First-order $L^{1}$-convergence for relaxation approximations to conservation laws. Comm. Pure Appl. Math. 51 (1998), no. 8, 857-895. MR1620220 (99f:65133)

[41] Wang, W.C.; Xin, Z., Asymptotic limit of initial-boundary value problems for conservation laws with relaxational extensions. Comm. Pure Appl. Math. 51 (1998), no. 5, 505-535. MR1604274 (99a:35172)

[42] Whitham, G. B., Linear and nonlinear waves, John Wiley \& Sons, New York, 1974. MR0483954 $(58: 3905)$

[43] Wang, W.-C., Nonlinear stability of centered rarefaction waves of the Jin-Xin relaxation model for $2 \times 2$ conservation laws. Electron. J. Differential Equations 2002, No. 57, 20 pp. (electronic). MR.1911924 (2003i:35183)

[44] Wang, W.-C.; Xin, Zhouping, Fluid-dynamic limit for the centered rarefaction wave of the Broadwell equation. J. Differential Equations 150 (1998), no. 2, 438-461. MR1658668 (99k:82066)

[45] Xin, Z., The fluid-dynamic limit of the Broadwell model of the nonlinear Boltzmann equation in the presence of shocks. Comm. Pure Appl. Math. 44 (1991), no. 6, 679-713. MR.1109376 (92f:76078)

[46] Xin, Z.; Xu, W. Q., Stiff well-posedness and asymptotic convergence for a class of linear relaxation systems in a quarter plane. J. Differential Equations 167 (2000), no. 2, 388-437. MR1793199 (2001j:35185)

[47] Xin, Z.; Xu, W.-Q., Initial-boundary value problem to systems of conservation laws with relaxation. Quart. Appl. Math. 60 (2002), no. 2, 251-281 MR1900493(2003f:35199)

[48] Xu, W.-Q., Boundary conditions and boundary layers for a multi-dimensional relaxation model. J. Differential Equations 197 (2004), no. 1, 85-117. MR2030150(2005b:35186)

[49] Xu, W.-Q., Relaxation limit for piecewise smooth solutions to systems of conservation laws. J. Differential Equations 162 (2000), no. 1, 140-173. MR.1741875 (2001i:35204)

[50] Yang, T.; Zhu, C., Existence and nonexistence of smooth solutions to $p$-system with relaxation, J. Differential Equations, 161 (2000), 321-336. MR1744146 (2000k:35174)

[51] Yong, W.-A., Singular perturbations of first-order hyperbolic systems with stiff source terms. J. Differential Equations 155 (1999), no. 1, 89-132. MR1693210 (2000c:35011)

[52] Yong, W.-A.; Zumbrun, K., Existence of relaxation shock profiles for hyperbolic conservation laws. SIAM J. Appl. Math. 60 (2000), no. 5, 1565-1575. MR1761762 (2001b:35208)

[53] Zeng, Y., Gas dynamics in thermal nonequilibrium and general hyperbolic systems with relaxation. Arch. Ration. Mech. Anal. 150 (1999), no. 3, 225-279. MR1738119 (2000k:35173)

[54] Zhu, C. J., Asymptotic behavior of solutions for $p$-system with relaxation. J. Differential Equations 180 (2002), no. 2, 273-306. MR1894014(2003d:35174) 